\title{
Attitude Control Analysis of Tethered De-orbiting
}

\author{
T. V. Peters, José Francisco Briz Valero, Diego Escorial Olmos, GMV, Spain \\ V. Lappas ${ }^{1}$, University of Patras, Greece \\ P. Jakowski, I. Gray, A. Tsourdos, Cranfield University, Cranfield, UK \\ H. Schaub, University of Colorado at Boulder, USA \\ R. Biesbroek, European Space Agency, Netherlands
}

\begin{abstract}
The increase of satellites and rocket upper stages in low earth orbit (LEO) has also increased substantially the danger of collisions in space. Studies have shown that the problem will continue to grow unless a number of debris are removed every year. A typical active debris removal (ADR) mission scenario includes launching an active spacecraft (chaser) which will rendezvous with the inactive target (debris), capture the debris and eventually deorbit both satellites. Many concepts for the capture of the debris while keeping a connection via a tether, between the target and chaser have been investigated, including harpoons, nets, grapples and robotic arms. The paper provides an analysis on the attitude control behaviour for a tethered de-orbiting mission based on the ESA e.Deorbit reference mission, where Envisat is the debris target to be captured by a chaser using a net which is connected to the chaser with a tether. The paper provides novel insight on the feasibility of tethered de-orbiting for the various mission phases such as stabilization after capture, de-orbit burn (plus stabilization), stabilization during atmospheric pass, highlighting the importance of various critical mission parameters such as the tether material. It is shown that the selection of the appropriate tether material while using simple controllers can reduce the effort needed for tethered deorbiting and can safely control the attitude of the debris/chaser connected with a tether, without the danger of a collision.
\end{abstract}

\subsection{Introduction}

The increase of satellites and rocket upper stages in low earth orbit (LEO) has also increased substantially the danger of collisions in space. Studies have shown that the problem will continue to grow unless a number of debris are removed every year [1, 2]. A typical active debris removal (ADR) mission scenario includes launching an active spacecraft (chaser) which will rendezvous with the inactive target (debris), capture the debris and eventually deorbit both satellites. Many concepts for the capture of the debris while keeping a connection between the target and chaser have been investigated, including harpoons, nets, grapples and robotic arms [310]. Towing the inactive target via a tether, however, seems to be the most promising idea which has been investigated for ADR missions, where the chaser can tow the debris, in a controlled and stable manner, in order to perform a controlled re-entry manoeuvre to the Earth's atmosphere without risk of casualties on ground [2-9].

The dynamics and control of two bodies connected via a tether using multiple or single attachment points is challenging with many aspects still unexplored. In a paper by Jasper and Schaub [10], the authors studied the tether dynamics and continuous open-loop thrust input shaping to attenuate the violent dynamics of TSS and hence avoid the collision between the end bodies. This approach, however, can be challenging due to the discrete on/off thruster

1 Corresponding author, Professor in Aerospace Systems, email: vlappas@upatras.gr 
capabilities. Several discrete thrust input shaping techniques are studied in [11]. These approaches are more realistic for on-off thrusters and offer better performance with respect to a step input in terms of end bodies collision avoidance and target attitude motion. The target's angular rate during tethered towing is studied in [12], however, the target's attitude has not been analyzed in detail. Investigation of the target's attitude is important in an active debris removal (ADR) mission scenario in order to avoid a tether wrapping up around the target and thus avoiding possible in-orbit collisions. The authors in [10] also emphasized the influence of the tether parameters, such as length, Young's modulus and damping ratio, on the system dynamics. Aslanov and Yudintsev [14] analyze the rotational motion of the target, when constant low thrust is applied by the active spacecraft. The study reveals that initial target orientation or initial slack in the tether can lead to tether tangling around the target which can result in tether rupture thus creating new debris in orbit. The study, however, does not consider closed-loop control of the chaser and its attitude motion impact on the target rotation. Following a deorbit burn, closed loop control of the chaser's attitude and relative position with respect to the target is analyzed in Ref [13]. Closed-loop control is advantageous due to the increased safety achieved by avoiding the collision between the end bodies and robustness for uncertain target mass and inertia properties. Nevertheless, closed-loop control adds complexity to the system, requires careful consideration of sensor performance and may increase the control effort due to sensor noise. Multiple control techniques for various tether based ADR maneuvers, debris capture with nets have been recently developed which tackle various control challenges with advanced control techniques such as sliding mode control, input shaping and others [27-31]

The paper provides an analysis on the attitude control behaviour for a tethered de-orbiting mission based on the ESA e.Deorbit reference mission [9] where Envisat is the debris target to be captured by a chaser using a net which is connected to the chaser with a tether. The various mission phases of post debris capture, debris/chaser tethered dynamics are analysed when towing takes place, in order to find the best design choices for the thrusters, tether (material, length, strength, controller bandwidth) and analyse their impact on the coupled tether-chaserdebris attitude stability, in order to ensure that the debris can be towed/de-orbited safely without colliding with the chaser.

\subsection{Active Debris Removal Mission Design}

Space Debris poses a significant problem for satellites and astronauts in orbit. With the number of space debris increasing, there is a renewed effort to develop technical solutions, methods and analyse possible techniques to capture, tow and perform controlled re-entry of space debris objects which pose the largest danger for in-orbit collisions. Many agencies, institutions are currently investigating how debris can be removed from low earth orbit (LEO). In this work, the European Space Agency e.Deorbit active debris removal (ADR) mission study is used as a reference mission, to study the attitude control implications of towing a large satellite such as Envisat using a tether. The e.Deorbit mission concept has been studied by several actors, including ESA, see [3-10]. The objective of the e.Deorbit mission is to to remove Envisat from orbit. All studies investigated several concepts for capturing the target, including robotic arms, tentacles, nets and harpoons. The nets and harpoons imply a flexible connection or tether between the chaser and the target. The tether transmits the force generated by the main thrusters on the chaser to the target in order to provide the $\Delta \mathrm{V}$ required for de-orbiting. The most recent e.Deorbit studies need to take into account the fact that Envisat has experienced an increase in angular velocity to about $3.5 \%[8,9]$. This implies that the first action the chaser must take after capture (either by harpoon or net), is to stabilize the target and reduce the angular velocity to zero. Various studies are currently investigating how to capture Envisat with an angular velocity of up to $5 \% \mathrm{~s}[9,10]$. A component of the towing part of the missions which is currently being heavily analysed is the tether. Although there is significant heritage on the use of tethers in space, it's material and dynamical behaviour when connected to two large bodies while 
significant amounts of thrust are being applied to the coupled system are unknown and complex [7-12]. Figure 1 shows the overall configuration of Envisat, including the definition of the body fixed reference frame axis directions. The origin of the body reference frame is at the interface plane of the spacecraft interface ring. Table 1 shows the mass properties, dimensions and orbit parameters of Envisat. Table 1 shows the orbital parameters of Envisat in September 2015 [13]. It is expected that by the time the mission is launched the orbital altitude will have decayed somewhat.

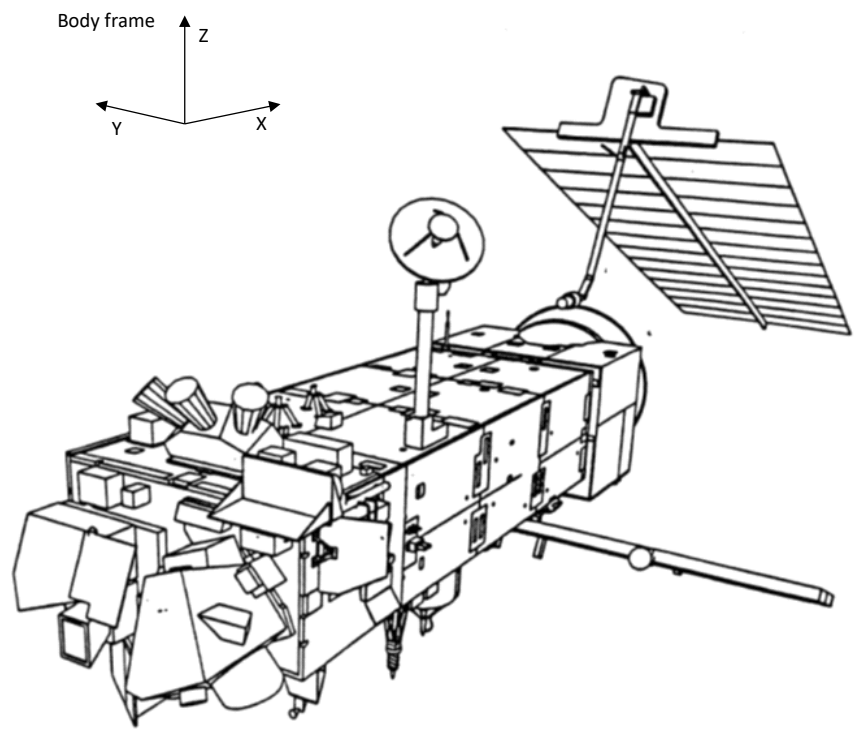

Figure 1: Envisat body frame

Table 1: Envisat properties

\begin{tabular}{lc}
\hline \multicolumn{1}{c}{ Parameter } & Value \\
\hline \hline Mass $[\mathrm{kg}]$ & 7827.867 \\
$\mathrm{I}_{\mathrm{xx}}, \mathrm{I}_{\mathrm{yy}}, \mathrm{I}_{\mathrm{zz}}\left[\mathrm{kgm}^{2}\right]$ & $(17023.3,124825.7,129112.2)$ \\
$\mathrm{I}_{\mathrm{xy}}, \mathrm{I}_{\mathrm{yz}} \mathrm{I}_{\mathrm{zx}}\left[\mathrm{kgm}^{2}\right]$ & $(397.1,344.2,-2171.1)$ \\
CoM $(\mathrm{x}, \mathrm{y}, \mathrm{z})[\mathrm{m}]$ & $(-3.905,-0.009,0.003)$ \\
Dimensions (body) $[\mathrm{m} \mathrm{x} \mathrm{m} \mathrm{x} \mathrm{m]}$ & $10.02 \times 2.75 \times 1.6$ \\
Dimensions (Solar panel) $[\mathrm{m} \mathrm{x} \mathrm{m} \mathrm{x} \mathrm{m]}$ & $14.028 \times 4.972 \times 0.01$ \\
Length $[\mathrm{m}]$ & 26.024 \\
Eccentricity & 0.000117 \\
Inclination $\left(^{\circ}\right)$ & 98.3274 \\
Perigee height $(\mathrm{km})$ & 765 \\
Apogee height $(\mathrm{km})$ & 766 \\
RAAN $\left(^{\circ}\right)$ & 303.2 \\
Argument of perigee $\left(^{\circ}\right)$ & 81.03 \\
\hline
\end{tabular}

The work from [13] collects and analyses available Envisat attitude data from different kind of observations (optical, Satellite Laser Ranging and Radar measurements) from the end of life of the satellite on April 8, 2012, and from simulations performed. Since then, the attitude of the satellite has experienced important changes, but several facts can be assumed:

i. Consistency of observation data and models: There is qualitative matching between radar measurements and SLR measurements but the high rotation rate measured does not fit the predicted rotational state with models and the cause is currently unknown. Several causes are considered such as a micro-meteoroid impact or energy release from the non-passivated ENVISAT. 
ii. Current rotation state: The main attitude motion corresponds to a relatively high rotation between $2 \%$ and $3.5 \%$ around the body z-axis (as defined in Figure 1 ). There are also smaller rotation components around the other body axes. The spin axis of the satellite is quite stable (within the radial coordinate system, which is fixed to the orbit) and, according to SLR measurements (from [14] is pointing in the direction opposite to the normal vector of the orbital plane in such a way that the spin axis makes an angle of $61.86^{\circ}$ with the nadir vector and $90.69^{\circ}$ with the along-track vector.

iii. Long term evolution: SLR measurements indicate that the spin period is increasing in time by $36.7 \mathrm{~ms} /$ day (according to measurements from [14]). Numerical simulations described in [13] accounting for gravity gradient as the dominating disturbance torque but excluding all damping torques show that gravity gradient stabilisation cannot be expected in the medium term (10 years). Numerical simulations described in Error! Reference source not found. include magnetic eddy current damping and find increases in the spin period of an order of magnitude comparable to that found in [14]. An extrapolation using an exponential fit to the SLR observation data indicates that the rotation rate may drop below $0.4 \%$ setween 2026 and 2028. Simulations that include gravity gradient and magnetic eddy current torques indicate that a transition to libration around a gravity gradient stabilised attitude starts at $0.4 \%$. The transition to a gravity gradient may occur before 2035. Because this estimate is based on an extrapolation, this value should be taken as highly uncertain.

In Ref [8], ESA is currently studying 3 different rotation scenarios during the e.Deorbit phase B1 project. These scenarios are:

i. Scenario 1

a. Spin axis in body frame is aligned with the + Ys axis.

b. Spin axis in LVLH frame is aligned with the $+\mathrm{H}$-bar axis.

c. Spin rate is $3.5 \%$ s.

ii. Scenario 2

a. Spin axis in body frame is along a direction contained in the YsZs plane at 45 degrees with respect to $+Y$ s and $+Z s$.

b. Spin axis in LVLH frame is aligned with the $+\mathrm{H}$-bar axis.

c. Spin rate is $5 \%$.

iii. Scenario 3

a. Spin axis in body frame is aligned with the $+Z s$ axis.

b. Spin axis in LVLH frame is at an angle of 45 degrees with respect to the $+\mathrm{H}-$ bar axis and is contained in the $\mathrm{H}$-bar/R-bar plane.

c. Spin rate is $5 \%$ s.

In the analyses performed in this paper, it is assumed that Envisat initially rotates at an angular velocity of $5 \%$ around the body y axis, and that the angular velocity of Envisat is aligned with the $\mathrm{H}$-bar axis.

\subsection{Chaser Parameters}

Table 2 shows the design characteristics of various chaser spacecraft developed and studied by different companies in e.Deorbit. It is envisaged that the chaser will be launched from Kourou by the Vega launch vehicle, with Soyuz as a back-up option. This is the reason why the mass of the chaser is bound to approximately $1500 \mathrm{~kg}$. Also included in the table are the flexible link capture methods, the force provided by the main thruster, the perigee altitude, the disposal $\Delta \mathrm{V}$ required for de-orbiting and the number of burns used to provide the disposal $\Delta \mathrm{V}$. The disposal $\Delta \mathrm{V}$ has been estimated based on the perigee height. Multiple burns are present in all designs to limit gravity losses associated with the de-orbiting sequence. The main thruster force tends to be either $850 \mathrm{~N}$, delivered by means of two $425 \mathrm{~N}$ bipropellant thrusters, or $1700 \mathrm{~N}$, delivered by means of four $425 \mathrm{~N}$ bipropellant thrusters. The main exception is the ELV design, which uses the AVUM main engine of $2540 \mathrm{~N}$ to perform the de-orbit burn. 
Table 2: e.Deorbit chaser designs [8-10]

\begin{tabular}{|c|c|c|c|c|c|c|}
\hline Design & $\begin{array}{c}\text { chaser } \\
\text { mass } \\
(\mathrm{kg})\end{array}$ & $\begin{array}{l}\text { Capture } \\
\text { method }\end{array}$ & $\begin{array}{c}\text { Main } \\
\text { thrust (N) }\end{array}$ & $\begin{array}{l}\text { Perigee } \\
(\mathbf{k m})\end{array}$ & $\begin{array}{c}\text { disposal } \Delta V \\
\left(\mathrm{~ms}^{-1}\right)\end{array}$ & $\mathbf{N}$ burns \\
\hline ESA CDF & 1570.5 & net & 850 & 40 & 216.13 & $3+1$ \\
\hline $\begin{array}{l}\text { ESA / ADS AGADR } \\
\text { low }\end{array}$ & 1500 & N/A & 200 & N/A & N/A & $\begin{array}{l}\text { multiple } 20 \\
\text { min burns }\end{array}$ \\
\hline $\begin{array}{l}\text { ESA / ADS AGADR } \\
\text { high [10] }\end{array}$ & 1500 & N/A & 2000 & $\mathrm{~N} / \mathrm{A}$ & N/A & $\begin{array}{l}\text { multiple } 20 \\
\text { min burns }\end{array}$ \\
\hline GMV AGADR & 1610 & $\mathrm{~N} / \mathrm{A}$ & 850 & $\mathrm{~N} / \mathrm{A}$ & N/A & multiple \\
\hline ADS & 1589.4 & net & 1700 & 70 & 190 & 3 \\
\hline ELV 1 & 1828 & net & 50 & 0 & 1260 & 63 \\
\hline ELV 2 & 1828 & net & 2540 & 0 & 223 & 2 \\
\hline TAS & 1592.8 & harpoon & 1600 & 40 & 216 & $3-4$ \\
\hline $\mathrm{KT}(\mathrm{OHB})$ & 1800 & net & 1600 & 30 & 220 & $6+1$ \\
\hline
\end{tabular}

Figure 2 shows the baseline chaser for the work presented in this paper. The chaser has been developed by Airbus Defense and Space for ESA for the e.Deorbit program [8, 9]. Figure 2 also shows the chaser spacecraft model including the body fixed frame. The origin of the body fixed frame is the centre of mass. The interface ring is the bottom of the cylinder connected to the main body. The platform design is based on a platform similar to the DEOS mission. In this image, the net launch canisters are the round light orange structures placed on the raised circular platform in the centre of the top face of the view on the left-hand side of Figure 2. Two net launch canisters are carried such that two capture attempts can be made. The chaser platform design is compact and has a low mass. The chaser design features 4 main engines of $425 \mathrm{~N}$ (two redundant engines), four intermediate, $220 \mathrm{~N}$ engines and twenty-four $22 \mathrm{~N}$ RCS engines [16]. In our study only two $425 \mathrm{~N}$ thrusters are used. The thruster models used in our simulation and system model are based on the Airbus bi-propellant 200/400N thrusters flown on ATV and have a Minimum Impulse Bit (MIB) capability of 0.09 Ns with an on time of 22-30 ms [26].
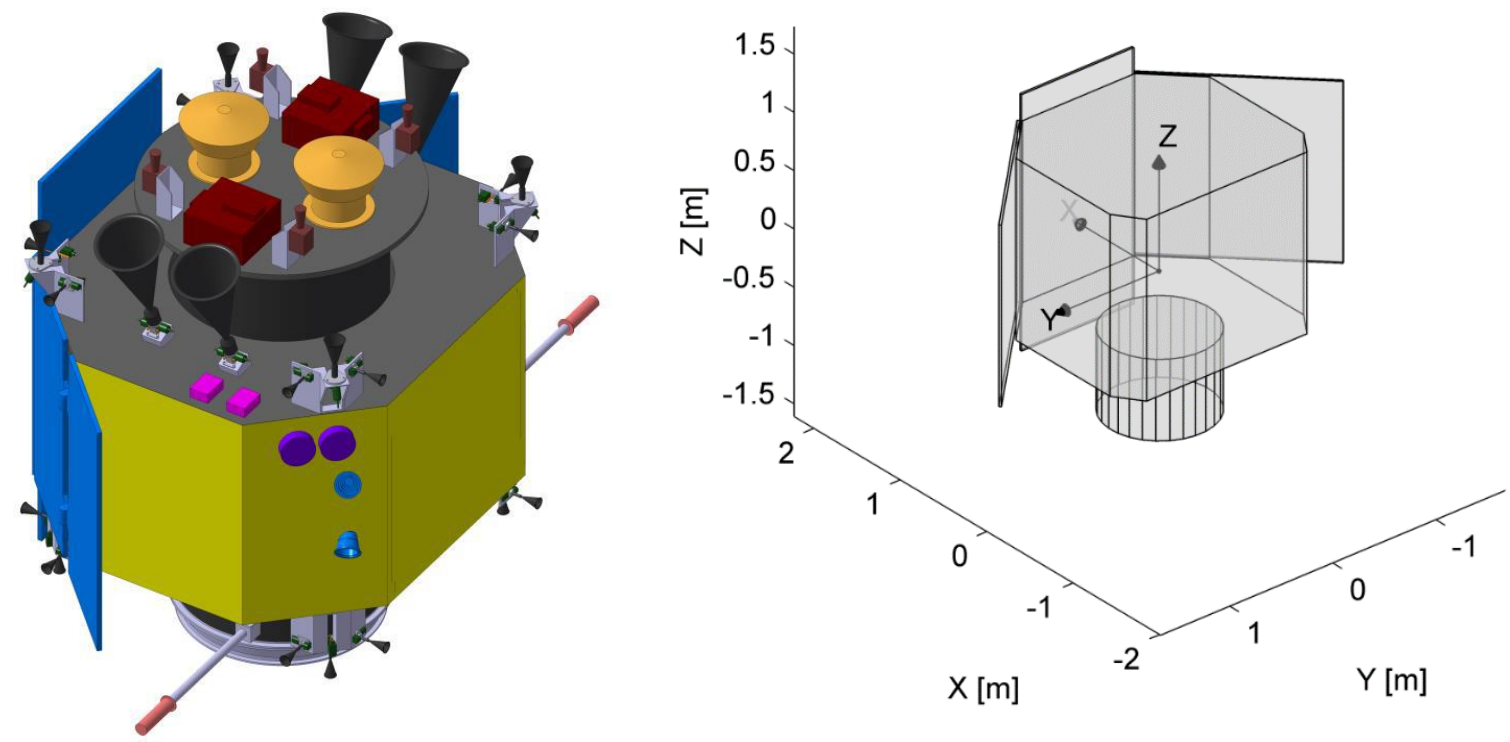

Figure 2: ADR chaser layout and body frame definition [9]

The operation of the thrusters is as follows: the $425 \mathrm{~N}$ thrusters operate in continuous mode only during the de-orbiting for the duration of the de-orbit burn, while the $220 \mathrm{~N}$ thrusters operate in 
pulse mode during the initial stabilization and the stabilization during and after the de-orbit burn. The $22 \mathrm{~N}$ thrusters are used in pulse mode for position and attitude control during all phases. Table 1 shows the mass properties of the chaser. The centre of mass location is defined with respect to the centre of the interface ring.

Table 3: Chaser mass properties

\begin{tabular}{lc}
\hline \multicolumn{1}{c}{ Parameter } & Value \\
\hline \hline Mass $[\mathrm{kg}]$ & 1610 \\
$\mathrm{I}_{\mathrm{xx}}, \mathrm{I}_{\mathrm{yy}}, \mathrm{I}_{\mathrm{zz}}\left[\mathrm{kgm}^{2}\right]$ & $1100,1160,450$ \\
$\mathrm{I}_{\mathrm{xy}}, \mathrm{I}_{\mathrm{yz}}, \mathrm{I}_{z x}\left[\mathrm{kgm}^{2}\right]$ & $0,0,0$ \\
$\mathrm{CoM}_{\mathrm{x}}[\mathrm{m}]$ & 0.01 \\
$\mathrm{CoM}_{\mathrm{y}}[\mathrm{m}]$ & 0 \\
$\mathrm{CoM}_{\mathrm{z}}[\mathrm{m}]$ & 1.18 \\
\hline
\end{tabular}

\subsection{Tether Characteristics}

Various tether designs, meaning lengths, material have been proposed in multiple studies [3-10]. The tether designs fall into two groups, soft tethers with a low stiffness, as proposed by ESA and stiff tethers, proposed by industry. The ESA e.Deorbit study proposes a Nylon tether with a length of $400 \mathrm{~m}$ and a stiffness of $132 \mathrm{~N} / \mathrm{m}$. Tethers designs proposed in the eDeorbit phase A studies are generally shorter and stiffer, with stiffness typically larger than $1000 \mathrm{~N} / \mathrm{m}$ [8]. Table 4 shows the properties of a number of high-strength materials that have been considered for application in space tethers in previous projects. Also included is rubber, which is an example of an elastic material. A synthetic fibre with similar or superior properties is spandex, also known as elastan. The e.Deorbit CDF study proposes a tether with a high elasticity (tether stiffness $k$ of $40-100$ $\mathrm{N} / \mathrm{m}$ ) and a length of $400 \mathrm{~m}$ [8]. The thrust level required for towing would be $800 \mathrm{~N}$. The AGADR study proposes a material with a Young's modulus of $0.01-0.1 \mathrm{GPa}$, i.e., comparable to rubber, and a tether length of $200 \mathrm{~m}$ [10]. The nominal tether stiffness would be $10 \mathrm{~N} / \mathrm{m}$. The deorbit burn would be performed with a $500 \mathrm{~N}$ engine.

Table 4: Properties of tether materials

\begin{tabular}{l|ccccccc}
\hline Material & $\begin{array}{c}\text { Density } \\
{\left[\mathrm{g} / \mathrm{cm}^{3}\right]}\end{array}$ & $\begin{array}{c}\text { Tensile } \\
\text { Strength } \\
{[\mathrm{GPa}]}\end{array}$ & $\begin{array}{c}\text { Young's } \\
\text { Modulus } \\
{[\mathrm{GPa}]}\end{array}$ & $\begin{array}{c}\text { Specific } \\
\text { strength } \\
{[\mathrm{kNm} / \mathrm{kg}]}\end{array}$ & $\begin{array}{c}\text { Ultimate } \\
\text { Elongation } \\
{[\%]}\end{array}$ & $\begin{array}{c}\text { Max. } \\
\text { temp } \\
{\left[{ }^{\circ} \mathrm{C}\right]}\end{array}$ & $\begin{array}{c}\text { Space } \\
\text { Qualified }\end{array}$ \\
\hline \hline Dyneema & 0.97 & 3.6 & 116 & 3711 & $3-4$ & 140 & YES2 \\
Zylon & 1.56 & 5.8 & 270 & 3718 & 2.5 & 600 & \\
Tecamid & 1.14 & 0.08 & 2.41 & 70 & 25 & 240 & \\
Kevlar & 1.44 & 3 & 112.4 & 2083 & 2.4 & 500 & \\
Nomex XF & 0.72 & - & - & - & - & 1100 & \\
Spectra & 0.97 & 3.68 & 133 & 3794 & 2.9 & - & SEDS/ TiPS \\
Twaron & 1.44 & 3.6 & 120 & 2500 & 4.4 & 500 & \\
Technora & 1.39 & 3.4 & 74 & 2446 & 4.5 & 500 & \\
Vectran & 1.41 & 3.2 & 72.4 & 2270 & - & 330 & \\
T1000G & 1.81 & 6.4 & 294 & 3536 & 2.2 & 1500 & \\
Rubber & 0.92 & 0.016 & $0.01-0.1$ & 16.3 & $200-400$ & $\sim 200$ & \\
\hline
\end{tabular}

Using the materials properties from Table 4, it is possible to determine what material corresponds best for the debris required to be towed. It is also possible to determine, whether the information and the assumed material can form a consistent and realistic set of assumptions for a tether. The parameters of interest are the stiffness $k$ and the tension in the tether. The tension cannot exceed the tensile strength of the material the tether is made of. The tether is 
assumed to behave like an elastic spring with spring constant $k$. The spring constant $k$ is related to the properties of the tether according to:

$$
k=\frac{E A}{l}
$$

Where $A$ is the cross-sectional area of the tether, $E$ is the modulus of elasticity of the tether material, $l$ is the length of the tether.

If the length and the elasticity are given and a material is assumed (i.e., a Young's modulus) then the cross sectional area of the tether can be calculated. The tension in the tether is equal to the force applied to the tether divided by the cross-sectional area.

$$
\sigma=\frac{F_{\text {teth }}}{A}
$$

The maximum force in the tether occurs during the de-orbit burn. Figure 3 shows a diagram of the de-orbit burn. The mass $m_{1}$ of the target, Envisat, is $8000 \mathrm{~kg}$. The mass $m_{2}$ of the chaser is $1400 \mathrm{~kg}$.

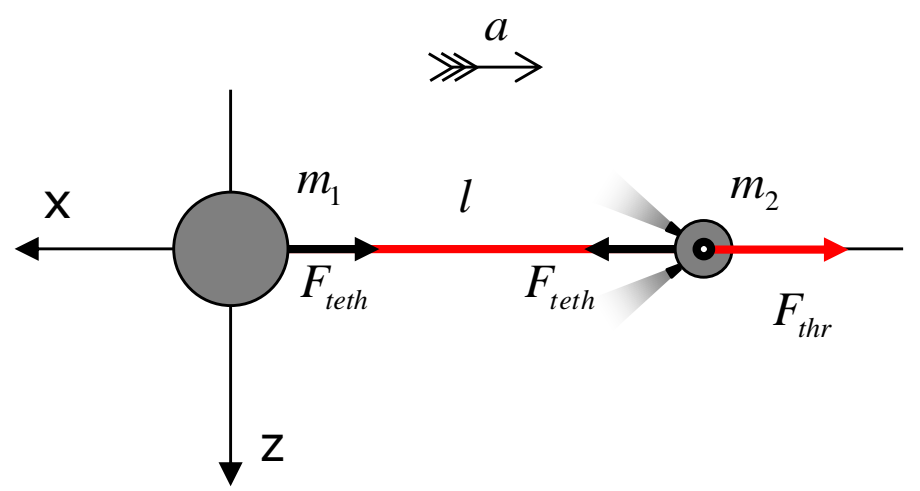

Figure 3: De-orbit burn model

The force in the tether under thrusting action is found by considering the steady state acceleration of both masses during the de-orbit burn. The thruster force accelerates both masses equally, such that the acceleration of the system is:

$$
a=\frac{F_{t h r}}{m_{1}+m_{2}}
$$

The force in the tether is then found by considering that the tether force needs to provide the target mass with acceleration a:

$$
F_{\text {teth }}=m_{1} a=\frac{m_{1}}{m_{1}+m_{2}} F_{t h r}
$$

That is, the force in the tether is equal to the thruster force multiplied by the ratio of the target mass to the total mass. Note that this is the steady state force in the tether. If the thrust is switched on abruptly, then the maximum force and the maximum tension in the tether can become much higher, depending on the material.

The AGADR results most closely resemble a tether made of rubber, which can have a Young's modulus of $0.1 \mathrm{GPa}$ as indicated in the study [10]. The AGADR study indicates a mass of $5 \mathrm{~kg}$ for the $200-\mathrm{m}$ tether, such that the cross-sectional area would be $27 \mathrm{~mm}^{2}$ if the tether has the density of rubber of $920 \mathrm{~kg} / \mathrm{m}^{3}$. The force in the tether is computed from Eq. (4), using a thruster force of $500 \mathrm{~N}$ resulting to a force in the tether of $425 \mathrm{~N}$, leading to a tension in the tether of $15.66 \mathrm{MPa}$, which is just below the ultimate tensile strength of rubber of $0.016 \mathrm{GPa}$. The 
tether stiffness can be computed from Eq (1) as $13.6[\mathrm{~N} / \mathrm{m}]$, which is of the same order of magnitude as the values stated in the AGADR study $(10 \mathrm{~N} / \mathrm{m})[10]$.

In summary, the e.Deorbit CDF study parameters are broadly consistent with a tether made of Nylon, while the AGADR study parameters are broadly consistent with a tether made of rubber. The e.Deorbit phase A results are consistent with the use of high strength materials such as Dyneema, Technora, Kevlar. In the current analysis, a 100-m tether made out of Dyneema is selected as the baseline design. The diameter of the tether is $1.5 \mathrm{~mm}$, leading to a stiffness of $2050 \mathrm{~N} / \mathrm{m}$.

\subsection{ADR Scenario Definitions and Assumptions}

This paper focuses on studying the attitude motion of the chaser-tether-target system for the critical parts of a tethered ADR mission, which are during and following a deorbit burn applied by an active chaser. A feedback control is developed to stabilize the tether and the target separation distance in a towing ADR mission. This improves safety substantially by reducing the danger of chaser-debris collision and thus fragmentation. The paper assumes that the challenging rendezvous and target capture has been already performed. The space debris is a passive, uncooperative satellite in low Earth orbit. The chaser is equipped with a large main engine $(\sim 2000 \mathrm{~N})$ for performing the deorbit burn and reaction control system (RCS) for applying force and torque corrections. The main engine is of an on-off type and the RCS can deliver variable thrust. Following the deorbit burn, the chaser activates two closed-loop controllers. First, the relative distance between the end bodies is controlled to maintain a small tension in the tether, and second, the chaser's orientation is controlled to ensure the correct attitude of the chaser. The end bodies are connected by a discretized viscous-elastic tether. Based on the attitude motion analysis $[18,19]$ of the inactive Envisat satellite, which is the focus of the e.Deorbit mission [17], a representative ADR mission scenario is considered which accounts for small, residual initial angular rates of the target prior to the deorbit burn. Furthermore, no input shaping of the deorbit burn is used. The main thrust is modeled as a step function, which accounts for simplified and worst-case approach. However, the reader should note that a combination of discrete deorbit burn shaping and closed-loop control following the deorbit burn may improve the performance. In the proposed analysis, the emphasis is placed on avoiding the tether tangling around the target which can result in tether rupture or debris collision and thus debris fragmentation.

The following section describes the ADR scenarios that are investigated in the current paper. Three scenarios are investigated:

- Stabilization after capture

- De-orbit burn (plus stabilization)

- Stabilization during atmospheric pass

The first two cases are studied by means of simulations, the third case is investigated analytically.

\subsubsection{Stabilization after capture}

The initial rotation rate of the target is $5 \% \mathrm{~s}$ around the body $y$-axis, which is aligned with the $y$ axis of the LVLH frame. The length of the tether is $50 \mathrm{~m}$. Table 5 shows the simulation test cases that will be studied. The frequency of the on-board software (OBSW) consistent with the operating frequency of LIDAR and camera sensors and the thrusters [26]. 
Table 5: Attitude Stabilisation test cases

\begin{tabular}{llccc}
\hline Case \# & \multicolumn{1}{c}{ Description } & Thrusters (N) & Tether stiffness (kN/m) & Frequency (Hz) \\
\hline \hline 1 & Nominal & $4 \times 220$ & 4100 & 5 \\
2 & RCS only & $4 \times 22$ & 4100 & 5 \\
3 & Low stiffness & $4 \times 220$ & 194 & 5 \\
4 & Low OBSW frequency & $4 \times 220$ & 4100 & 1 \\
\hline
\end{tabular}

During the initial stabilization phase the assist thrusters are used to ensure that the attitude motion of the target can be stopped. The assist thrusters are active during the first 700 seconds after capture.

\subsubsection{De-orbit burn}

The initial rotation rate of the target is $0.5 \%$ around the body $y$-axis. This value is lower than the $5 \%$ at the start of the stabilization after capture case, considering that the target has been successfully stabilised during the manoeuvre. The length of the tether varies from $100 \mathrm{~m}$ to 400 $\mathrm{m}$, and its stiffness varies from $100 \mathrm{~N} \mathrm{~m}^{-1}$ to $2050 \mathrm{~N} \mathrm{~m}^{-1}$.

Table 6: De-orbit burn test cases

\begin{tabular}{llcccc}
\hline Case \# & \multicolumn{1}{c}{ Description } & $\begin{array}{c}\text { Thrusters } \\
(\mathbf{N})\end{array}$ & $\begin{array}{c}\text { Tether } \\
(\mathbf{m})\end{array}$ & $\begin{array}{c}\text { Tether stiffness } \\
(\mathbf{k N} / \mathbf{m})\end{array}$ & $\begin{array}{c}\text { Frequency } \\
(\mathbf{H z})\end{array}$ \\
\hline \hline 1 & Nominal & $2 \times 425$ & 100 & 2050 & 5 \\
2 & High thrust & $4 \times 425$ & 100 & 2050 & 5 \\
3 & Long tether & $2 \times 425$ & 400 & 512 & 5 \\
4 & Long, soft tether & $2 \times 425$ & 400 & 100 & 5 \\
5 & Low frequency & $2 \times 425$ & 100 & 2050 & 1 \\
6 & Long soft tether low frequency & $2 \times 425$ & 400 & 100 & 1 \\
\hline \hline
\end{tabular}

The burn manoeuvre starts after 10 seconds. After 10 seconds the target rotates away from its initial attitude that is perfectly aligned with the tether. That is to say, waiting 10 seconds ensures a more realistic attitude at the start of the burn. The burn manoeuvre lasts 500 seconds and then a stabilization phase begins for 1000 seconds. The assist thrusters are active during the first 700 seconds after burning.

\subsection{Attitude Control ADR Model, Simulations and Analysis}

A tethered flight simulator is used for tethered de-orbiting. The simulator consists of a dynamics/kinematics environment (DKE), the on-board software (OBSW) and an output handling block. The simulator runs with a variable step-size in order to ensure proper simulation of the tether, avoiding singularities in the forces generated in the tether. A rate transition is included between the DKE and the OBSW to ensure that the DKE runs with a variable step size, and the OBSW runs at a fixed, user-defined frequency (for example, $1 \mathrm{~Hz}$ or $10 \mathrm{~Hz}$ ). This set-up ensures that the simulator as a whole is stable, while the on-board software runs at the correct frequency. The Simulink model used uses the rapid accelerator functionality of Simulink. By using the rapid accelerator, simulations of the order of 2000 seconds of simulated time with a tether stiffness of the order of $8000 \mathrm{~N} / \mathrm{m}$, a tether length of $50 \mathrm{~m}$ and a mass of the order of $150 \mathrm{~g}$ with a discretization of 9 segments can be run in under 5 minutes.

Figure 4 shows the dynamics model used to simulate the tether with the chaser spacecraft connected to a target spacecraft by means of a tether. The tether is simulated using the KelvinVoigt model, which uses discrete mass points connected by spring-damper elements that only generate force when the tether is in tension. That is to say, a tether element only generates force when the elongation of the element is larger than zero. All mass points are simulated in inertial space and experience the effect of the central gravity term plus $J_{2}$. No other disturbance forces 
terms are included. The chaser and the target of course experience the force of the tether. The attitude dynamics only includes the torque generated due to the offset of the tether attachment point from the centre of mass.

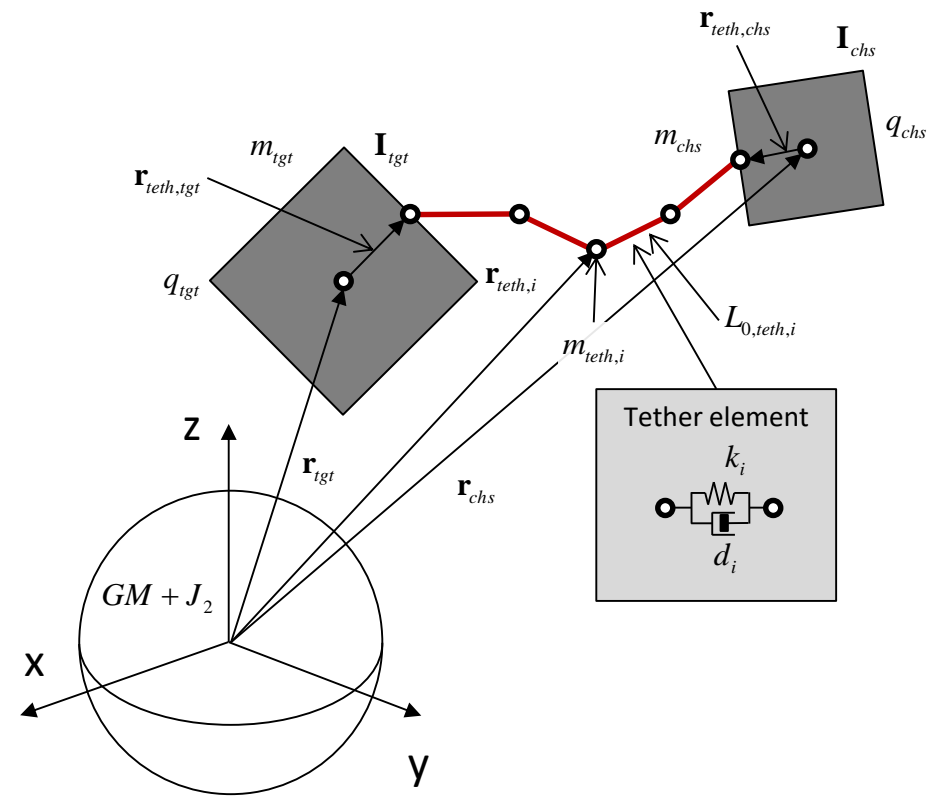

Figure 4: Tethered dynamics model

The following convention is used for the definition of the rotation angles:

- pitch: rotation around $y$-axis of the LVLH frame

- yaw: rotation around z-axis of the LVLH frame

- roll: rotation around $x$-axis of the LVLH frame

The tether can transmit forces, but it cannot transmit torques. This means that from the nominal position on the x-axis of the LVLH frame the chaser can control pitch and yaw motions of the target, but not the roll motion which would twist the tether.

\subsubsection{Equations of Motion}

The dynamics system considered in the paper consists of a chaser, debris and a tether connecting the two bodies, see Fig. 4 . Each of the end bodies is modeled as a rigid body which can translate and rotate, resulting in a 6 degrees of freedom model. The target (subscript 'tgt') is assumed to be a passive, inactive satellite. The chaser (subscript 'chs') has an active control system which is able to deliver both pure force and torque. The tether (subscript 'teth') is discretized in 2-point masses and 3 equidistant massless tether links for the model, however the fidelity can be increased to 10 discrete masses if necessary. Each tether element is modeled as a parallel springdamper system. When a given tether link length, $\boldsymbol{I}_{\mathbf{i}}, i=\{1-3\}$, is smaller than its natural length, $I_{0, \text { teth,i, }}$ the tether force in a given tether element vanishes. Discretized tether model has been widely used in the previous studies [9-13]. It accounts for transverse oscillations of the tether and adds only little complexity into the system. The effect of the number of the tether nodes on the behavior of the system has been studied in $[12,13]$. The number of the tether nodes greater than 2 does not change considerably the observed behavior of the system, but adds significant computation time. Translational and rotational motion of each end body is fully described by its inertial position in the ECl frame, $\boldsymbol{r}$, inertial velocity in the ECl frame, $\boldsymbol{V}$, attitude quaternion, $\boldsymbol{q}$, and angular rate vector in body frame, $\omega$. The attitude quaternion of each body is expressed as: 


$$
\boldsymbol{q}=\left[\begin{array}{llll}
q_{1} & q_{2} & q_{3} & q_{4}
\end{array}\right]^{T}
$$

where $\boldsymbol{q}_{4}$ denotes the quaternion scalar part. The attitude quaternion, $\boldsymbol{q}$, describes the satellite orientation with respect to the $\mathrm{ECl}$ frame. The angular rate vector of each body with respect to the $\mathrm{ECl}$ frame is expressed in a given body frame as $\boldsymbol{\omega}=\left[\begin{array}{lll}p & q & r\end{array}\right]^{T}$.

The kinematic relationships for each end body are given by:

$$
\begin{gathered}
\dot{\boldsymbol{r}}=\boldsymbol{V} \\
\dot{\boldsymbol{q}}=\frac{1}{2}\left[\begin{array}{ccc}
q_{4} & -q_{3} & q_{2} \\
q_{3} & q_{4} & -q_{1} \\
-q_{2} & q_{1} & q_{4} \\
-q_{1} & -q_{2} & -q_{3}
\end{array}\right]\left[\begin{array}{l}
p \\
q \\
r
\end{array}\right]
\end{gathered}
$$

The translational inertial acceleration of each end body is given by:

$$
m \ddot{\boldsymbol{r}}=-m \mu \frac{\boldsymbol{r}}{|\boldsymbol{r}|^{3}}+\boldsymbol{F}+\xi \boldsymbol{I}
$$

where all vectors are expressed in inertial frame. The first term on the right-hand side of Eq. (8) denotes the Earth gravitational force, $\boldsymbol{F}$ corresponds to the actuation force, $\boldsymbol{T}$ is a force applied by the tether link neighboring a given end body, and $\xi$ is a unit step function, defined for each tether element as:

$$
\xi= \begin{cases}1, l-l_{0}>0 \\ 0, l-l_{0} \leq 0\end{cases}
$$

The attitude dynamics of each end body is given by the Euler equation:

$$
\dot{\boldsymbol{\omega}}=\mathbf{I}^{-1}\left(M+M_{g g}+r_{i} \times \xi T-\omega \times \mathbf{I} \boldsymbol{\omega}\right)
$$

where all vectors are expressed in body frame. The first term in the parenthesis on the right-hand side of Eq. (10), $\boldsymbol{M}$, denotes the actuation torque, $\boldsymbol{M}_{g g}$ corresponds to the gravity gradient torque, third term denotes the torque generated by the neighboring tether link force, where $r_{i}$ is the displacement of the tether attachment points with respect to the center of mass ( $i=$ chs, teth, tgt), for the chaser and the target, respectively and the last term is the gyroscopic term. Note, that for the passive target, the actuation torque is $\boldsymbol{M}=0$.

\section{Tether Dynamics}

Using the time derivative of the magnitude of the tether link length vector, $\boldsymbol{l}_{i}$ the tether nodes translational dynamics equations are given by:

$$
m \ddot{\boldsymbol{r}}=-m \mu \frac{\boldsymbol{r}_{\iota}}{\left|\boldsymbol{r}_{\iota}\right|^{3}}+\xi_{\iota} \boldsymbol{T}_{\iota}+\xi_{\iota+1} \boldsymbol{T}_{\iota+1}
$$




\section{Closed-Loop Control}

The attitude control model designed, uses a position control function that is included as a double PD controller, that operates on the elongation and the in-plane vertical and out-of-plane deviation from its current position, plus a PD controller on the LVLH position. The attitude control function is also a PD controller, based on the quaternion error and the angular velocity. Both controllers are described in the following section.

The chaser has an active guidance and control system which allows it to control its position and attitude. During the deorbit burn, the chaser performs the attitude control required to align the chaser in its LVLH frame, to ensure the thrust is applied in correct direction. An attitude PD controller from [22] has been used. Its performance is satisfactory, achieving zero Euler angle orientation in the chaser's LVLH frame with a $0.1^{\circ}$ accuracy. The analysis of the attitude controller during the deorbit burn is not the focus of this study, and will not be studied in subsequent sections. Following the deorbit burn, the chaser controls the separation distance with respect to the target and the chaser's attitude. Two feedback controllers are used: a linear separation distance PID controller and a linear tether heading controller. The closed-loop control is a means to reduce the risk of the tether tangling around the target and it increases the robustness for initial target angular rates. Relatively simple guidance and control schemes are considered in this work and detailed analysis of their performance is beyond the scope of this paper.

Our study does not include navigation or sensor noise concerns. Furthermore, this paper considers the continuous force and torque variations to be perfectly implemented. This means that no thrusters dynamics are included. When a higher fidelity mission scenario is considered, control implementation limitations such as pulse width modulation and minimum impulse bit need to be addressed. For the scope of this paper such noise inclusions and thruster dynamics would mask some subtleties of the dynamics being studied. Sensing the relative motion, and the impact of the sensor noise on the closed loop performance is beyond the scope of this note.

\section{Relative Distance PID Controller}

In previous studies $[9-10,15,16]$, it is shown that the slack in the tether should be avoided for safety considerations. When the tether is slack, the target is allowed to move freely, which can be dangerous for numerous reasons. First, slack in the tether results in more violent dynamics and reduces the closest approach of the end bodies which increases the probability of collision and thus fragmentation. Secondly, free rotation of the target can lead to tether tangling and eventually its rupture. For those reasons, the relative distance control law is developed which maintains a small residual tension in the tether.

The relative distance error, $e$, is given by:

$$
e=l_{0}+\Delta l+\left|\boldsymbol{r}_{c h s}\right|+\left|\boldsymbol{r}_{t g t}\right|-|\Delta \boldsymbol{r}|
$$

where $l_{0}$ is the natural length of the tether, $\Delta l$ is a design parameter corresponding to the desired tether elongation, and $\Delta r=r_{c h s}-r_{t g t}$ is the distance between the chaser and the target centers of mass. Differentiating Eq. (7) with respect to time yields:

$$
\dot{e}=-\left(V_{c h s}-V_{t g t}\right)^{T} \frac{\Delta \boldsymbol{r}}{|\Delta \boldsymbol{r}|}
$$


Next, the commanded magnitude of the force is given by a simple proportional, derivative and integral control law:

$$
\boldsymbol{F}_{\text {comm }}=k_{P} e+k_{D} \dot{e}+k_{1} \int_{0}^{t} e \mathrm{dt}
$$

Finally, the commanded thrust obtained in Eq. (11) is applied in the direction of the relative distance between the chaser and the target, which gives:

$$
\boldsymbol{F}_{\text {comm }}=F_{\text {comm }} \frac{\Delta \boldsymbol{r}}{|\Delta \boldsymbol{r}|}
$$

\section{Tether Heading Feedback Control}

Following the deorbit burn, the attitude of the chaser is controlled, so that it is aligned with the tether. For both satellites, the alignment angle, $\theta_{\mathrm{C}}$, is defined as the angle between the tether attachment point vector and the tether line. Thus, for the chaser, the desired attitude is determined by the vectors $\boldsymbol{r}_{\mathrm{i}}$ and $-\Delta \boldsymbol{r}$ being parallel, and hence the desired chaser's alignment angle equal to null. This configuration ensures that the tether does not apply any torque to the chaser.

The attitude quaternion error, $q_{\mathrm{e}}$, is defined as:

$$
\boldsymbol{q}_{e}=\left[\begin{array}{c}
\hat{\boldsymbol{e}}_{\Delta \theta} \sin \frac{\theta_{C}}{2} \\
\cos \frac{\theta_{C}}{2}
\end{array}\right]
$$

where $\hat{\boldsymbol{e}}_{\Delta \theta}$ is a unit vector denoting the rotation axis around which the chaser must be rotated. Since the chaser's attitude is controlled relatively to the tether, the chaser will track the direction of the tether. When this approach is combined with the relative distance control, it is evident that no global position and attitude control of the chaser is performed. The whole system will tend to oscillate or tumble around the nadir direction, achieving stable gravity gradient configuration [10]. Since the orientation of the whole system varies very slowly in time, the desired angular rate of the chaser is set to around all body axes as $\boldsymbol{\omega}_{\text {ref }}=0$.

The Lyapunov attitude control law from [21] has been simplified to a PID control law, neglecting the gyroscopic term and external, known torque. The known external torque can be approximated by a torque applied by a tether, which would require accurate computation of the tether force. On the other hand, the gyroscopic term is proportional to the square of the chaser's angular rate which is approximately equal to the orbital rate, hence the gyroscopic term is negligible as well. This gives the simplified attitude PID control law:

$$
\begin{gathered}
\boldsymbol{T}_{c o m m}=\boldsymbol{K} \boldsymbol{q}_{e, 1: 3} \operatorname{sign}\left(q_{e, 4}\right)-\widetilde{\boldsymbol{P}} \boldsymbol{\omega}_{C}+\widetilde{\boldsymbol{K}}_{I} \int_{0}^{t} \boldsymbol{q}_{e, 1: 3} \operatorname{sign}\left(q_{e, 4}\right) \mathrm{dt} \\
\tilde{\boldsymbol{P}}=\boldsymbol{P}+\boldsymbol{P} \boldsymbol{K}_{I} \mathbf{I}, \quad \tilde{\boldsymbol{K}}_{I}=\boldsymbol{K} \boldsymbol{P} \boldsymbol{K}_{I}
\end{gathered}
$$


where $\operatorname{sign}(\cdot)$ is the signum function which ensures the shortest distance to the reference attitude.

Therefore, as shown in Eq's, 6-18, the position and attitude control are decoupled.

\subsubsection{Stabilization after capture}

In this section the ADR simulation results for the scenarios presented in Table 5 are presented. Figure 5 shows a typical simulation image of the stabilization scenario. The tether is shown in blue, with mass points represented as dots. The chaser trajectory is shown in red (zoomed in frame in Fig. 5). In this simulation, the chaser moves along a small 'figure 8 ' trajectory. In this nominal stabilization case, all thrusters are used ( $220 \mathrm{~N}$ and $22 \mathrm{~N}$ RCS thrusters).

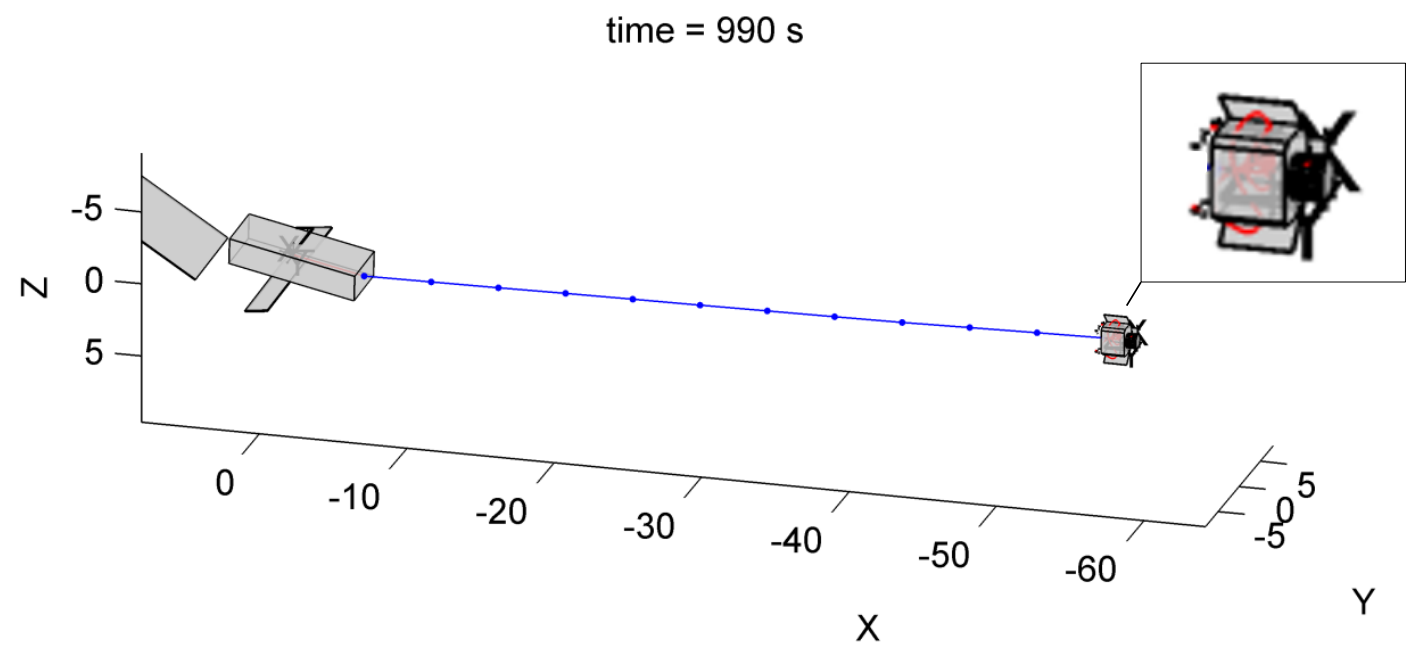

Figure 5: Simulation of ADR stabilization scenario

Figure $6 a$ shows the tension in the tether. The tension remains well below the maximum tension allowed for Dyneema.
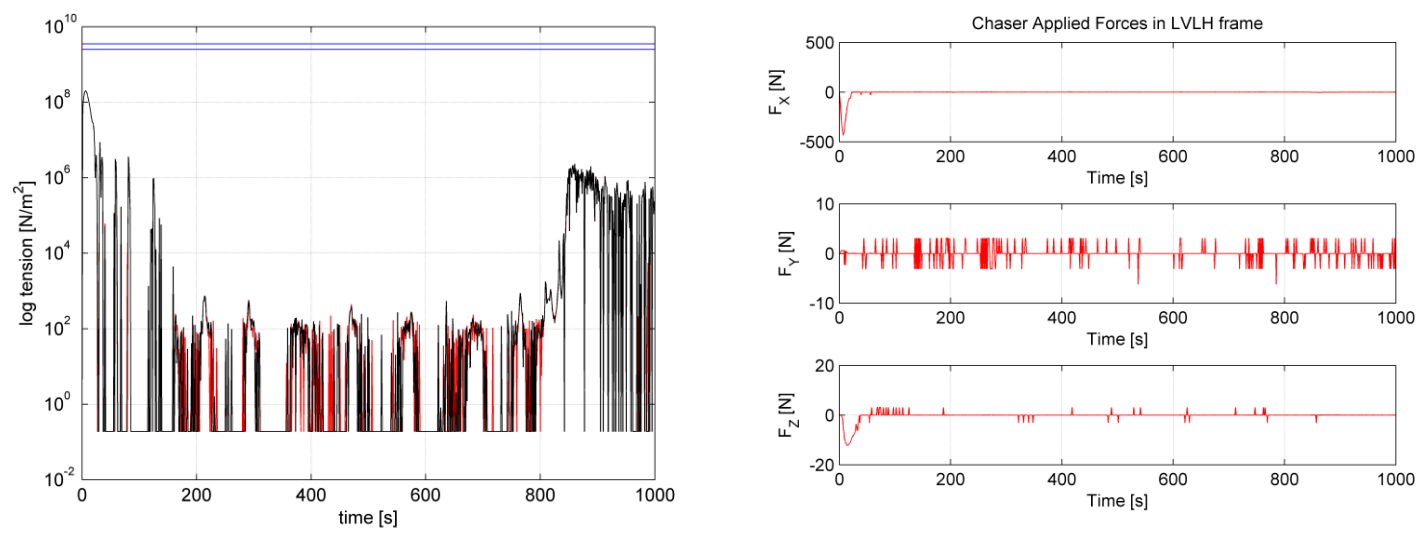

Figure 6: (a) Tether tension, nominal case (b) ADR Chaser forces in LVLH frame, nominal case

Figure $6 \mathrm{~b}$ shows the chaser thruster forces in the LVLH frame. The maximum force during the initial stabilization is about $440 \mathrm{~N}$, which is about half of the maximum allowable force (which is generated if all four $220 \mathrm{~N}$ assist thrusters are firing). Figure 7 shows the attitude of the target and the attitude rates. The target starts with an angular velocity of $5 \% \mathrm{~s}$, which is brought to zero within $20 \mathrm{~s}$. The maximum deviation along the pitch axis is $50^{\circ}$. 

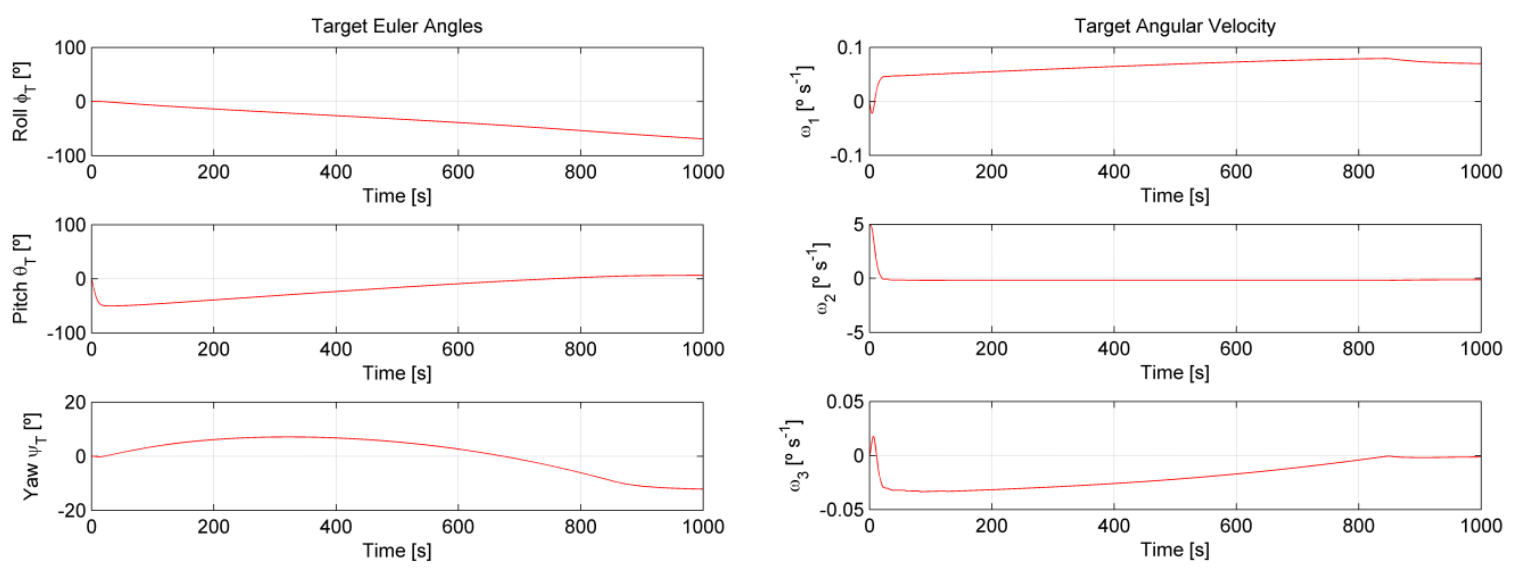

Figure 7: Target attitude and attitude rates, nominal case

The next simulation presented is the case in which only RCS thrusters are used (22N). Figure 8 shows a simulation still taken at the moment that the rotation rate of Envisat is brought to zero. The simulation image shows that Envisat rotates more than $90^{\circ}$ from the original orientation. Envisat rotates approximately $100-110^{\circ}$ before it stops rotating.

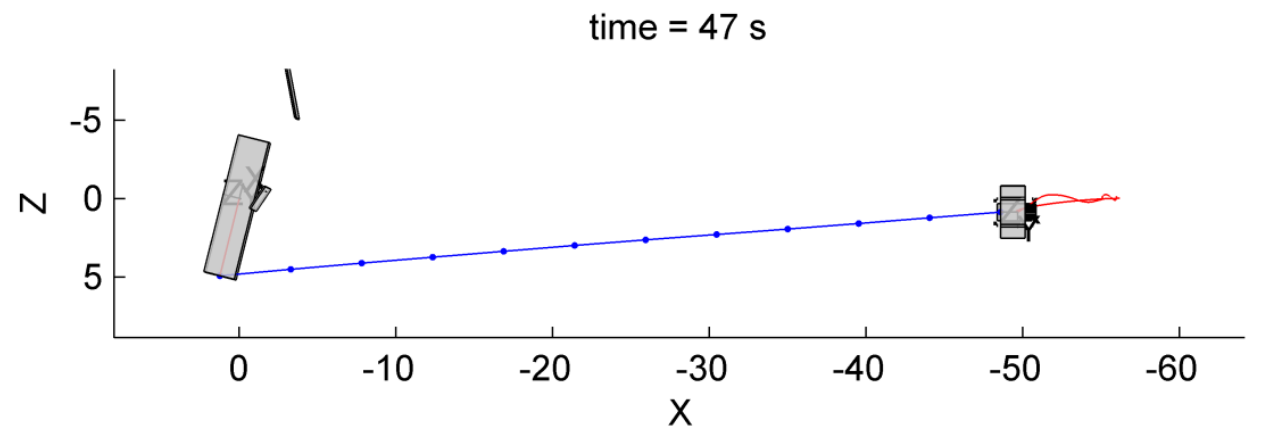

Figure 8: Simulation still at the moment the rotation rate is brought to zero
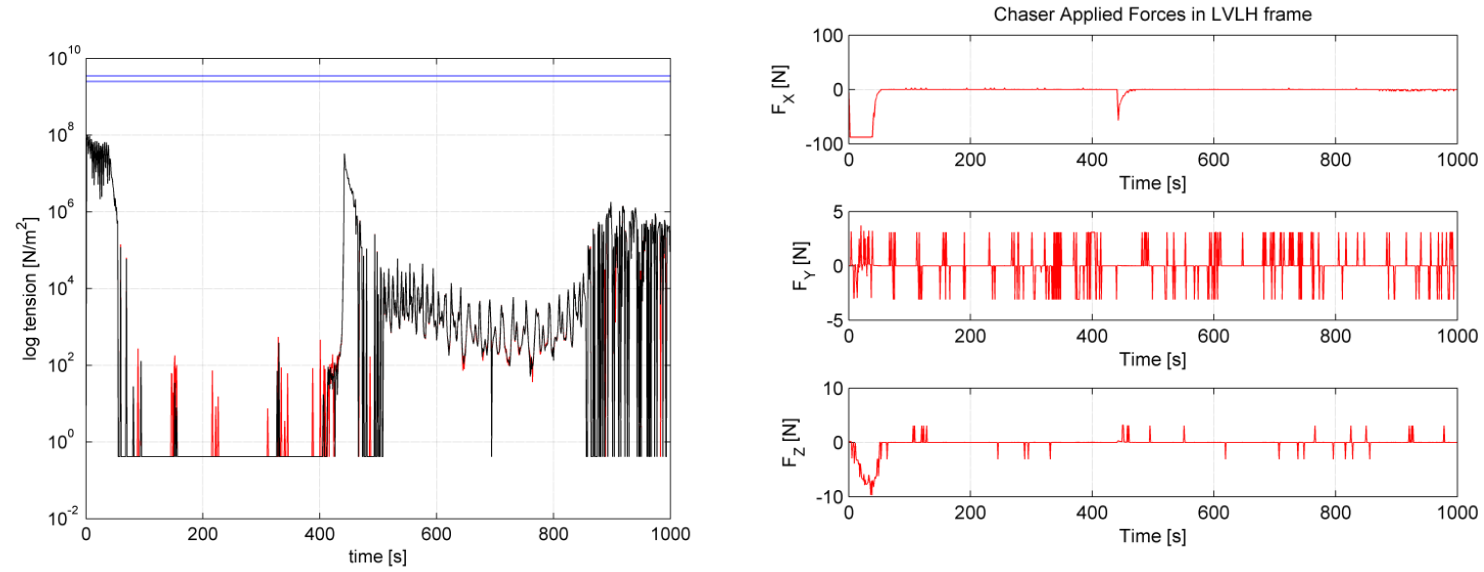

Figure 9: (a) Tether tension (b) Chaser forces in LVLH frame - RCS only case

Figure 9a shows the tension in the tether for the RCS thrusters only case. As expected the maximum tension in the tether is lower than in the nominal case. Figure $9 \mathrm{~b}$ shows that during the initial stabilization, the chaser applies the maximum force $(88 \mathrm{~N})$ in the x-direction of the LVLH frame, that is, the thrusters are saturated. Figure 10 shows the attitude of the target and the attitude rates. The target starts with an angular velocity of $5 \%$, which is brought to zero in the itch/yaw axes, in about $40 \mathrm{~s}$. The maximum angular deviation is difficult to establish from this plot, because the target simultaneously pitches, rolls and yaws. The maximum angle between the 
target body $x$-axis and the $x$ axis of the LVLH frame is $106^{\circ}$, which is in line with the attitude shown in the simulation still. The roll angle requires further control as it begins to diverge, however a slow roll spin can prove useful as it provides gyroscopic stability.
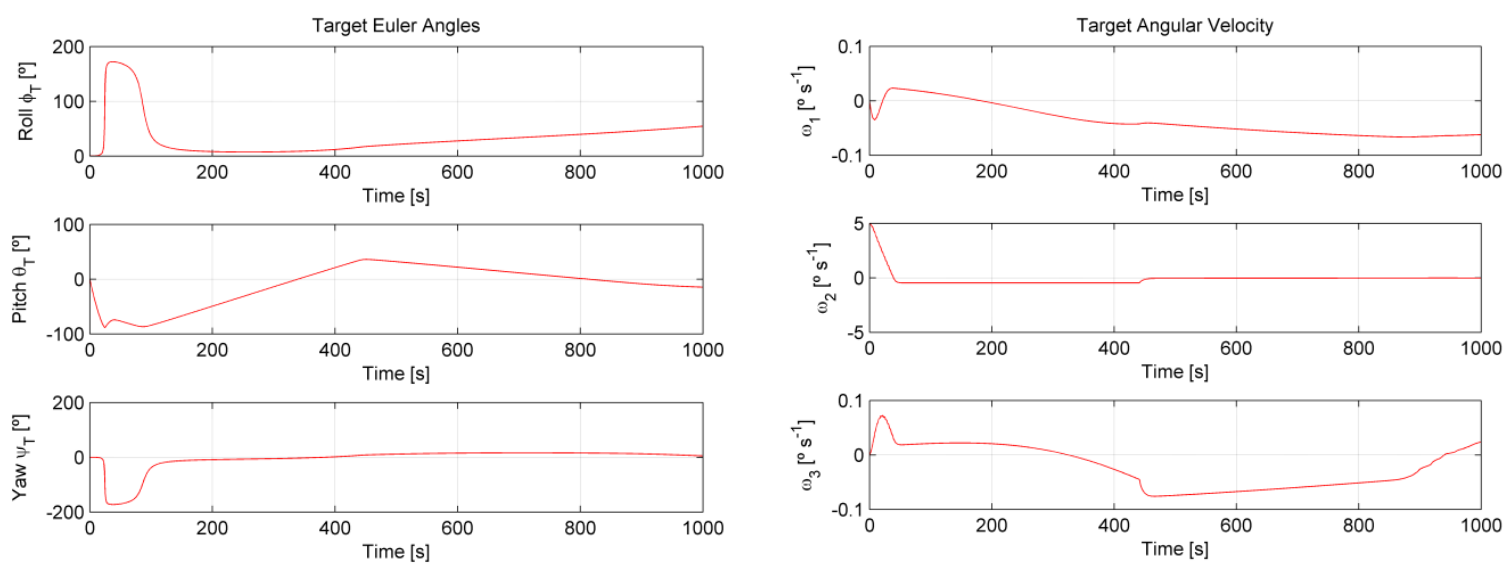

Figure 10: Target attitude and attitude rates, RCS only case

The next case to be investigated is for a low tether stiffness. Figure 11a shows the tension in the tether. The maximum tension in the tether is about the same as in the nominal case. Figure 11b shows that during the stabilization, the chaser needs to fire the RCS (assist) thrusters several times. The plot shows 8 distinct thruster activation events (that is, the thrust in the $x$-direction of the LVLH frame). This indicates that the stabilization takes a lot longer than in the nominal case.
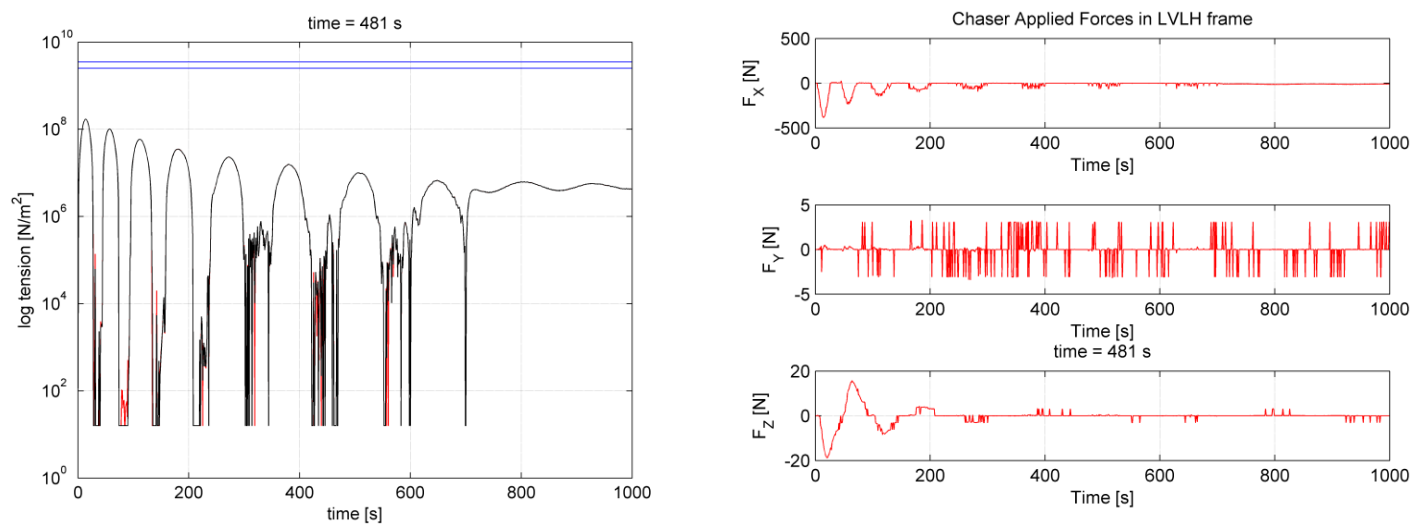

Figure 11: (a) Tether tension (b) Chaser forces in LVLH frame - low tether stiffness case

Figure 12 shows the attitude of the target and the attitude rates. The target starts with an angular velocity of $5 \%$. The stabilization shows considerable overshoot. The maximum angular deviation is about $60^{\circ}$, or about $10^{\circ}$ larger than in the nominal case. The target attitude overshoots several times. It should be noted that the controller gains were not modified between the nominal case and the low tether stiffness case, such that the controller behaviour could potentially still be improved. On the other hand, it should be noted that the decreased stiffness causes the desired force on the target to be applied with a delay and with lower precision when compared to the nominal stiffness: the tether needs to be extended considerably before it starts delivering the desired force on the target. This means that it takes longer to apply a force on the target and the chaser would need to move further away from its original position to achieve the same force when compared to the nominal tether stiffness.

Figures 13, 14 show the low frequency simulation scenario. Figure $13 \mathrm{~b}$ shows that during stabilization the thrusters are much more active, and that the actuation signals are much noisier. 

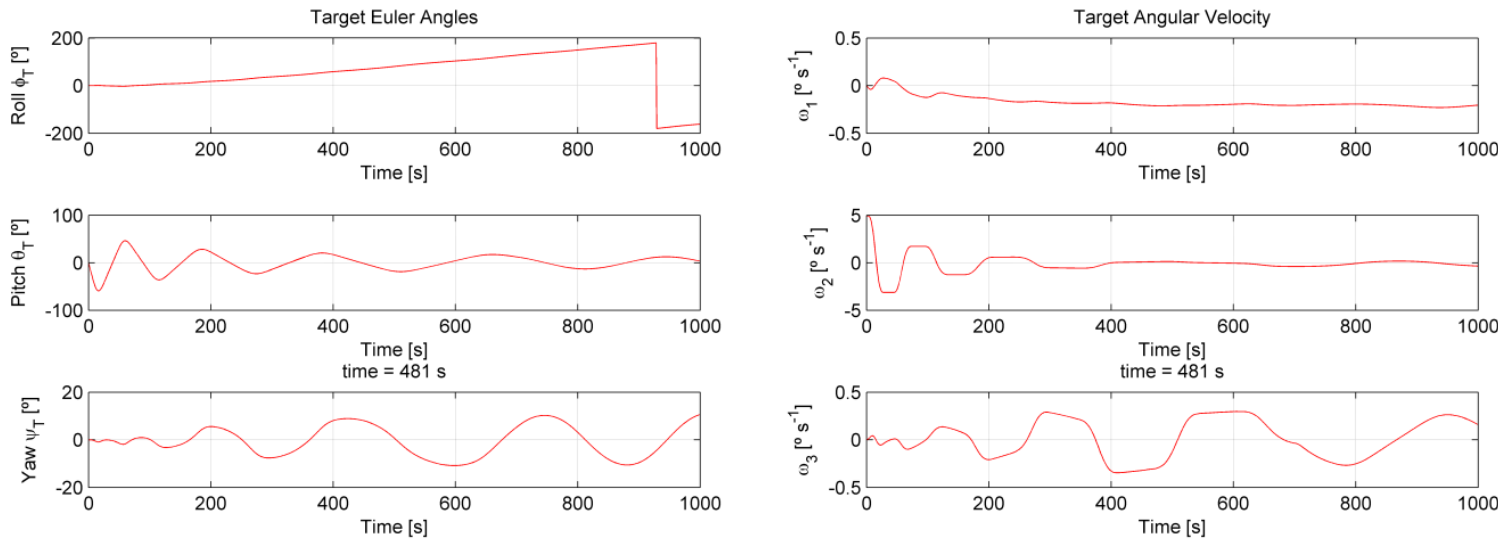

Figure 12: Target attitude and attitude rates, low tether stiffness case
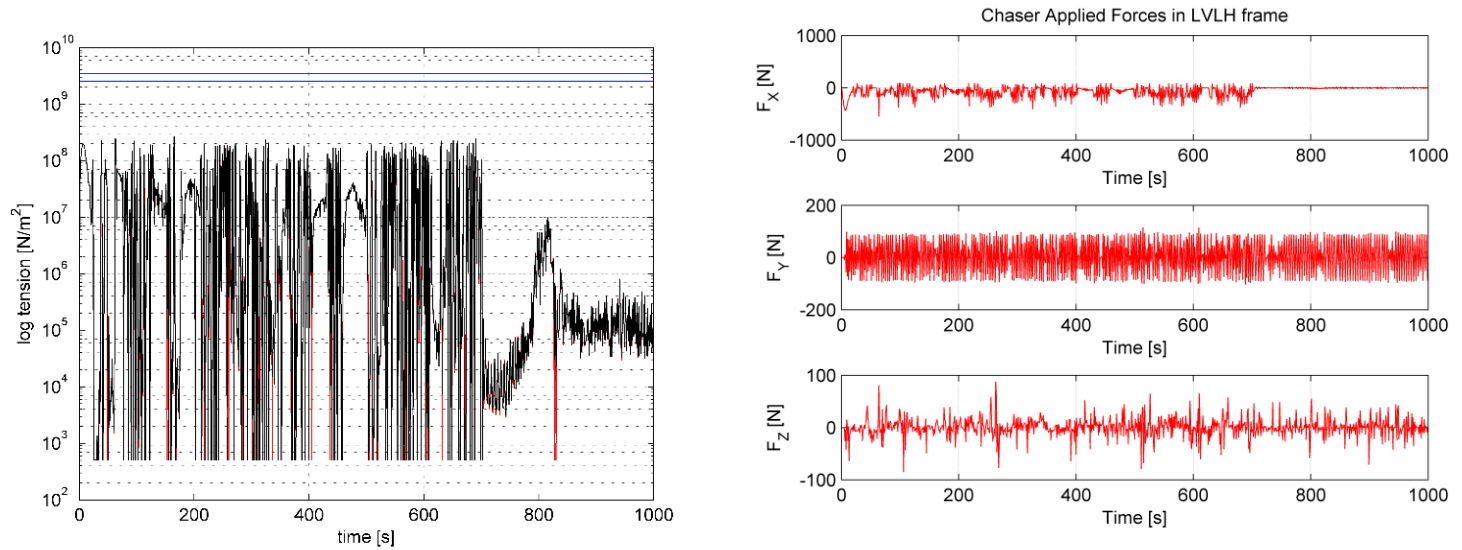

Figure 13: (a) Tether tension (b) Chaser forces in LVLH frame - low OBSW frequency case

Figure 13a shows the tension in the tether. The maximum tension in the tether is about the same as in the nominal case. The tether tension stays at a high level for far longer than in the nominal case, which indicates that the controller is not able to bring down the rotation rate as effectively as in the nominal case. Figure 14 shows the attitude of the target and the attitude rates. The target starts with an angular velocity of $5 \%$. The maximum angular deviation is $50^{\circ}$, which is the same as in the nominal case. The target attitude retains an oscillation with an amplitude similar to the maximum deviation until the assist thrusters stop being used at $700 \mathrm{~s}$. This indicates that the controller does not succeed in stabilizing the attitude of Envisat.
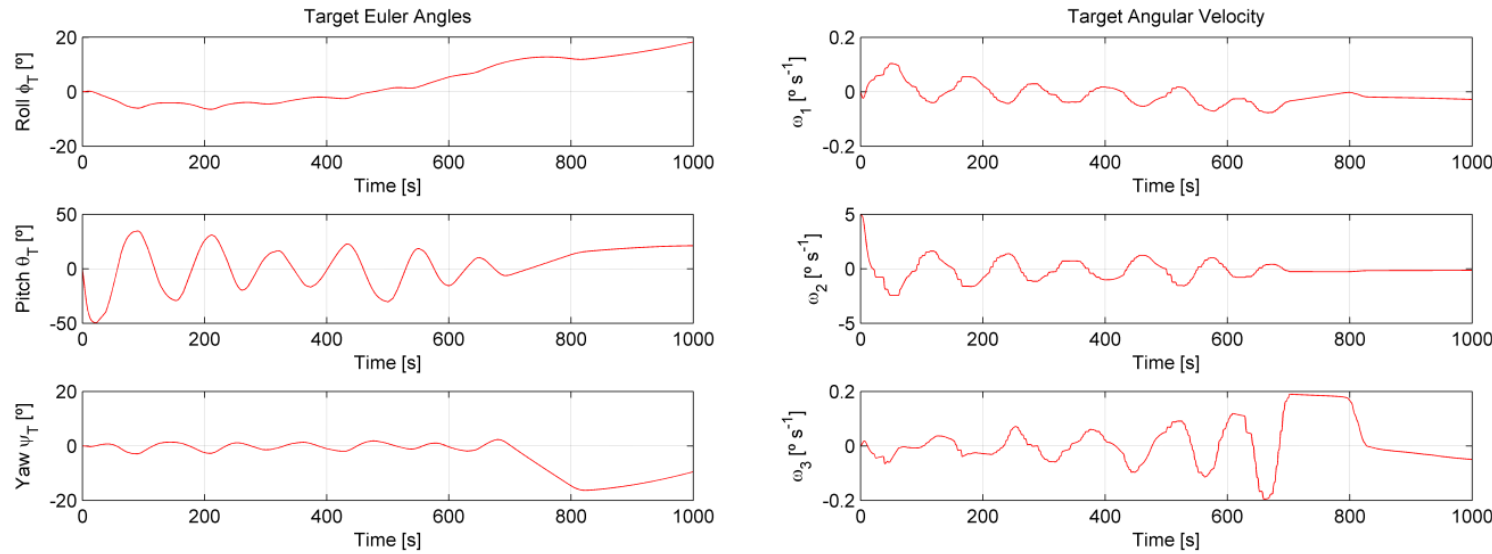

Figure 14: Target attitude and attitude rates - low OBSW frequency case 


\subsubsection{De-orbit Burn}

In this section the ADR simulation results for scenarios 1,5 and 6 presented in Table 6 , for the Deorbit burns. Figure 15a shows a slight overshoot at the beginning of the burn, followed by a constant tension profile until the end of the manoeuvre. Figure $15 \mathrm{~b}$ shows the forces applied to the chaser for the nominal case. The $x$-axis force shows a slight overshoot to stabilize the elongation of the tether, where the forces along the $y$ and $z$-axes are above the forces shown during the stabilization.
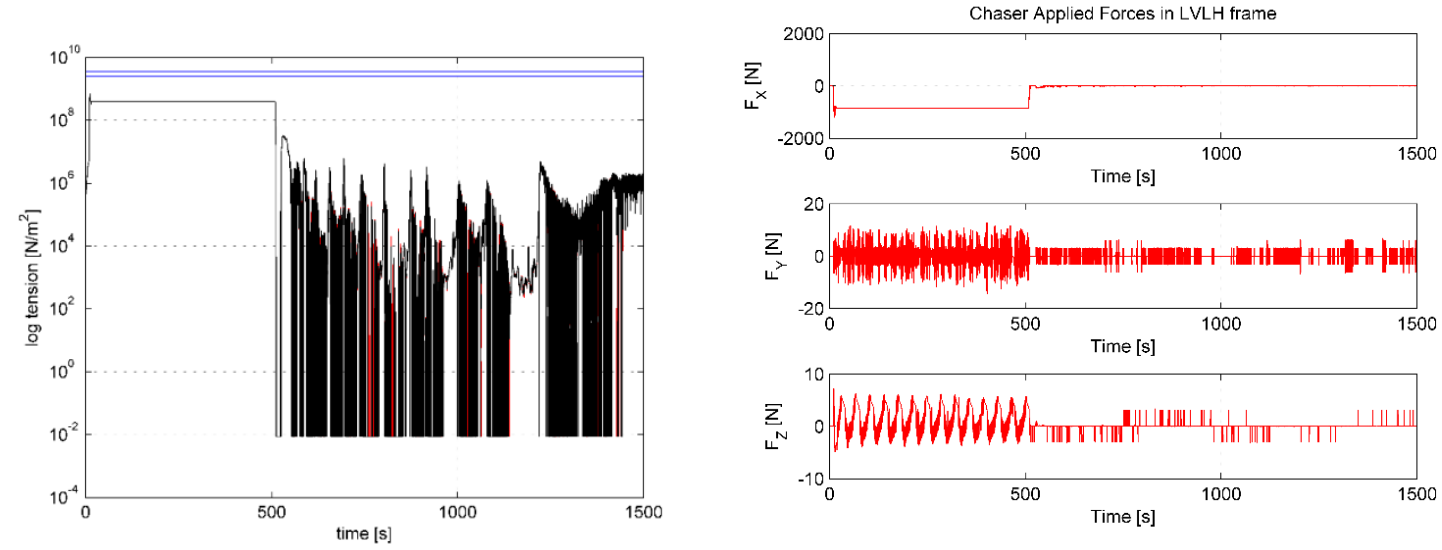

Figure 15: (a) Tether tension (b) Chaser applied forces in LVLH frame - nominal case

The tranquilization after the de-orbit burn shows a tension level similar to that of the tranquilization after the capture phase. Figure 16 shows a constant frequency low amplitude oscillation during the burning phase, caused by the constant force on the target. After the propelled manoeuvre, Envisat rolls, pitches and yaws at the same time, with an amplitude of $30^{\circ}$. Pitch and yaw are damped, using the same gains used in the stabilization after capture case, which could be further adjusted for this case. The current controller is not capable of controlling the roll motion.
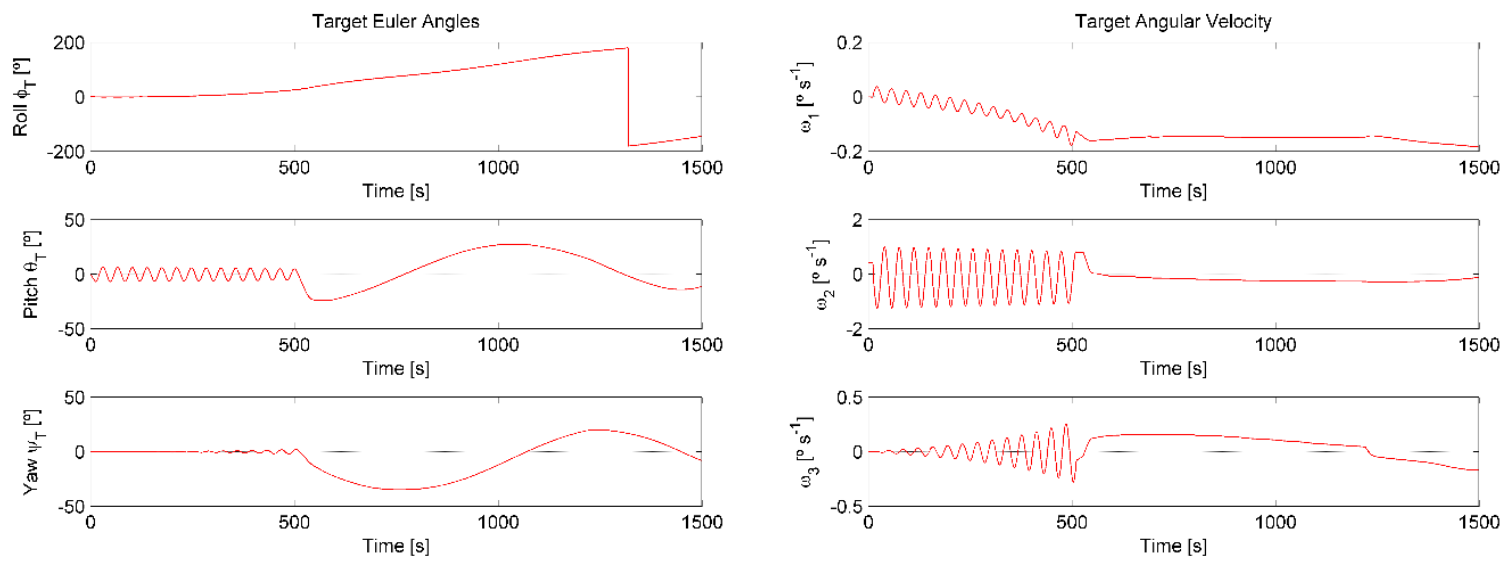

Figure 16: Target attitude and attitude rates, nominal case

Figure 17 shows in detail a full period of oscillation, close to 36 seconds. 


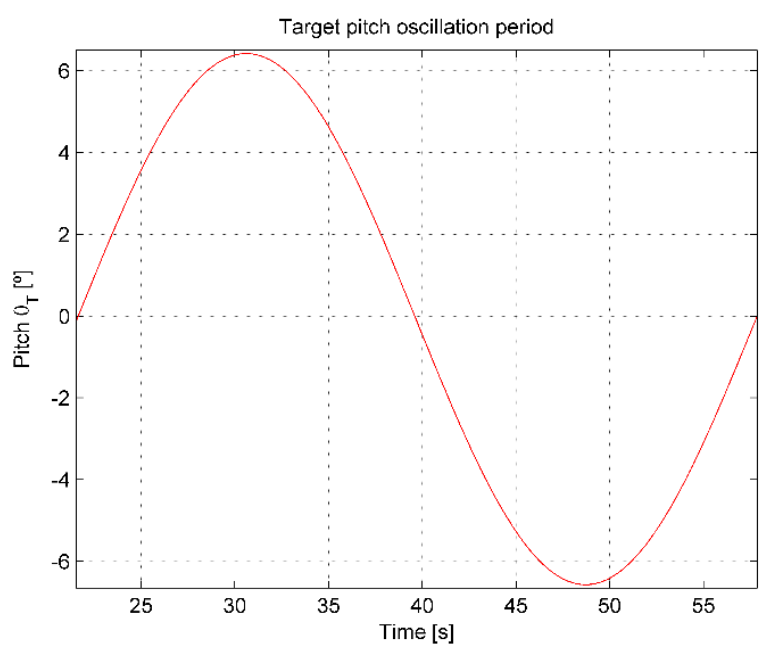

Figure 17: Target oscillation period

Figure 18 shows the position deviation when the OBSW frequency drops to $1 \mathrm{~Hz}$. The controller is not capable of following the changes in the chaser attitude. This causes the main thrusters to produce a resulting force out of the chaser-target line, which deviates the chaser from its equilibrium position. The motion of the chaser is still stable.

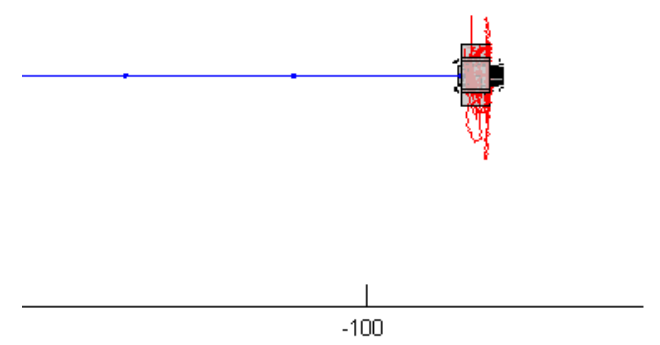

Figure 18: Chaser $\mathrm{X}-\mathrm{Z}$ movement in LVLH frame, low OBSW frequency case

Figure 19a shows that the tension is noisier as the chaser moves abruptly, but the maximum tension level remains below the maximum allowed. Figure $19 \mathrm{~b}$ shows that the $X$-axis forces are at the same level shown in the nominal case, but noisier. The $Y$ and $Z$ forces are higher as the chaser attitude changes the main thruster direction. The attitude control gains could be changed in this scenario to further improve the behaviour, as the gains are the same used for the nominal case. 

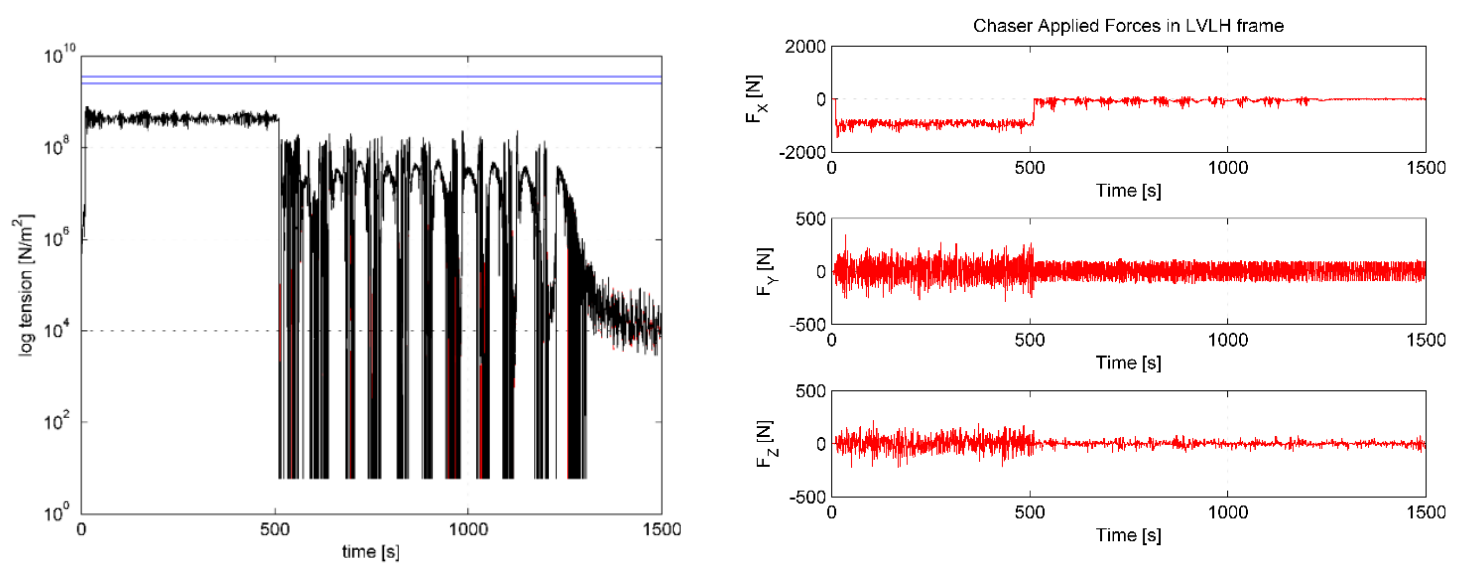

Figure 19: (a) Tether tension (b) Chaser applied forces in LVLH frame - low OBSW frequency

\section{case}

Figure 20 shows that the target attitude is contained amongst the maximum values obtained in the nominal case. The chaser behaviour does add a chaotic component to the target attitude, but the movement does not diverge.
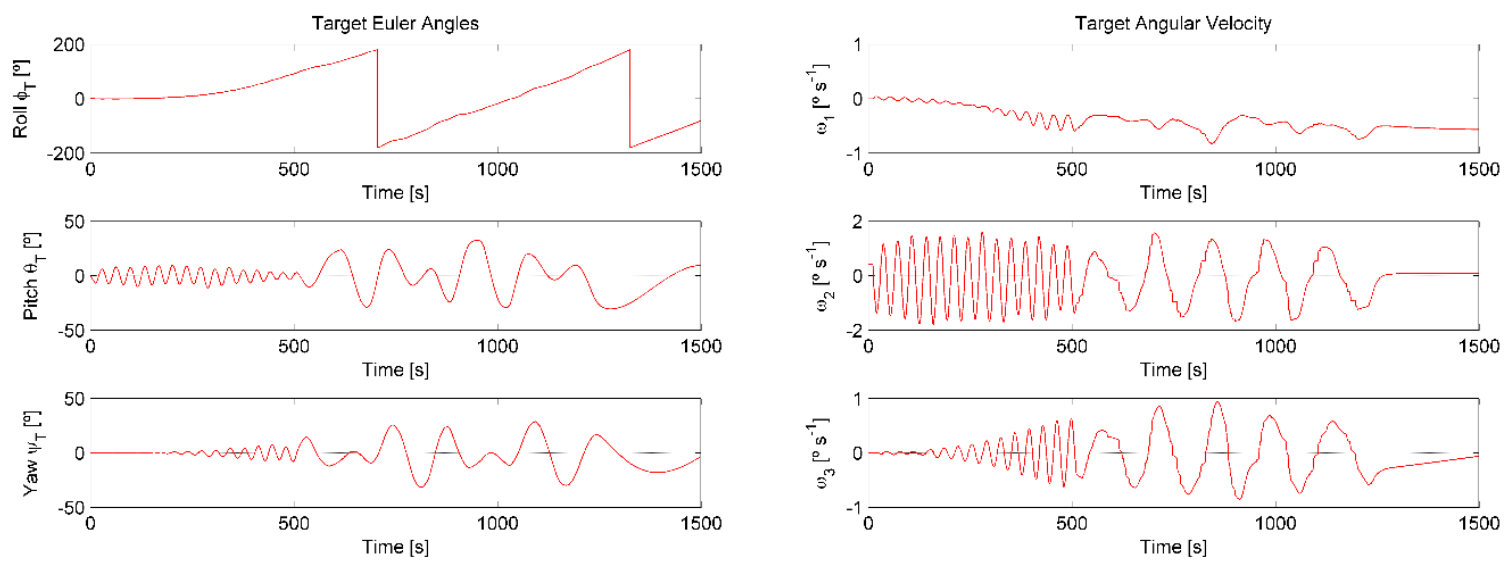

Figure 20: Target attitude and attitude rates, low OBSW frequency case

Figure 21 shows that the long soft tether operating at a $1 \mathrm{~Hz}$ frequency presents a combination of the problems of both long tether/low frequency scenarios. There is an overshoot at the beginning of the burning phase, followed by an abrupt behaviour of the chaser attempting to stay at the equilibrium point. 


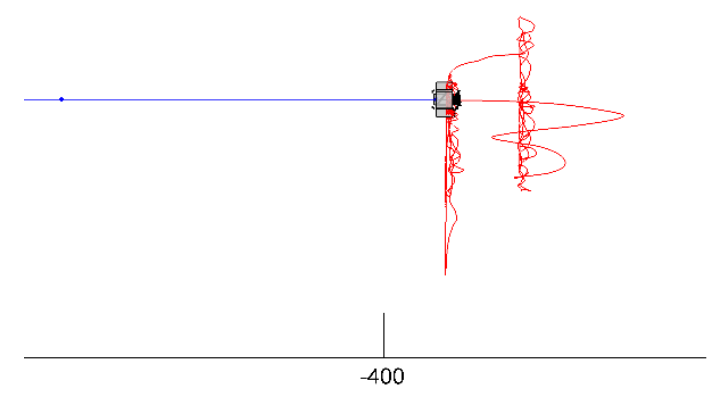

Figure 21: Chaser X-Z movement in LVLH frame, long soft tether low OBSW frequency case Figure 22a shows a tension overshoot at the beginning of the manoeuvre, followed by a noisy pattern with a constant mean tension level during the burn phase. Figure $22 \mathrm{~b}$ shows that the force profile is the same as observed during the low frequency case. The secondary control thrusters require a higher control force to compensate the initial elongation.
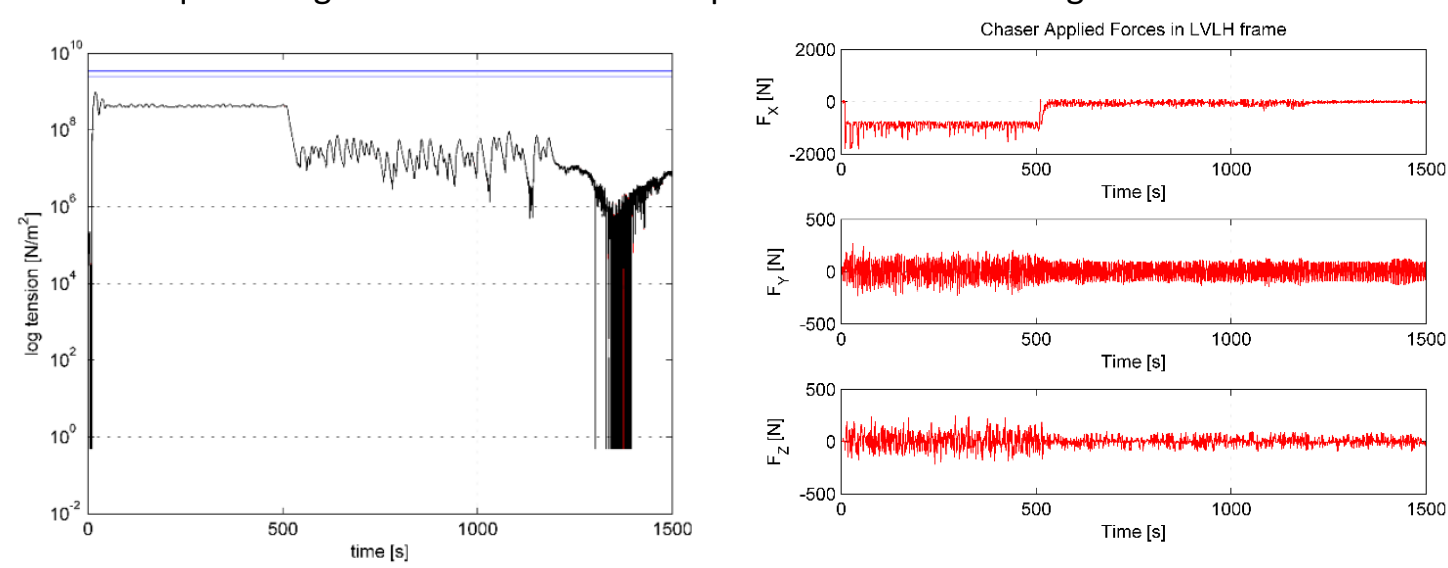

Figure 22: (a) Tether tension (b) Chaser applied forces in LVLH frame - long soft tether low OBSW frequency case

Figure 23 shows the target attitude angles and rates. The chaser behaviour does not negatively affect the debris attitude.
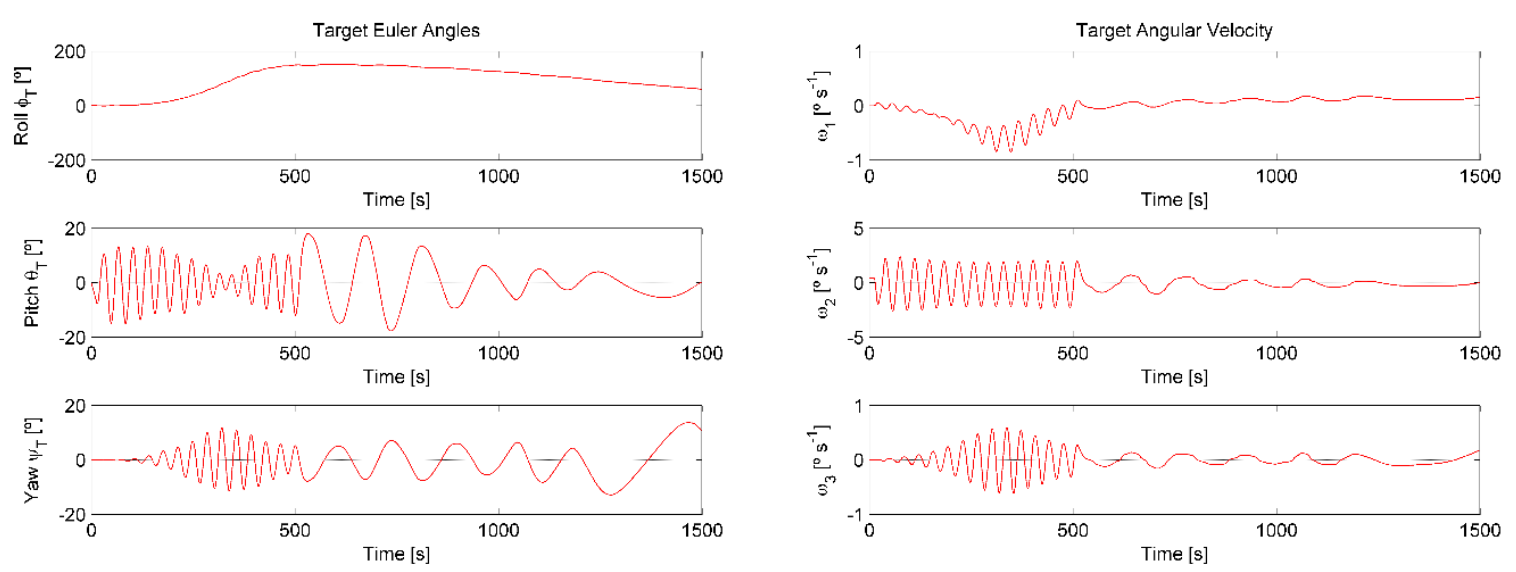

Figure 23: Target attitude and attitude rates, long soft tether low OBSW frequency case 


\subsubsection{Stabilization during Atmospheric Pass}

The pass through the atmosphere is conceptually similar to the de-orbit burns, or to a combination of the de-orbit burns and the stabilization. In this case, the aerodynamic attitude torques may cause a spin-up of the target or other unwanted attitude motion that the chaser needs to control. This is similar to the stabilization after capture. Furthermore, the ballistic coefficients of the chaser and the target may be different. The configuration would be such that the chaser is behind the target. Depending on the orientation, Envisat may present a large or a small surface area to the incoming stream. The drag force may therefore vary substantially. The situation would become problematic if the drag force on the target would become larger than the chaser can compensate for and the tether would go slack. That is, the chaser needs to be able to compensate for the differential drag force during the entire de-orbiting sequence.

The lowest perigee altitude before the final deorbit burn is $200 \mathrm{~km}$. The maximum atmospheric density at this altitude is $3.65 \cdot 10^{-10} \mathrm{~kg} / \mathrm{m}^{3}$. The velocity at perigee is equal to $8.1 \mathrm{~km} / \mathrm{s}$, leading to a dynamic pressure of $0.012 \mathrm{~Pa}$. In the maximum drag configuration, Envisat's body z-axis points in the direction of the flow. In the minimum drag configuration, Envisat's body $x$-axis points in the direction of the flow. In the ADS deorbiting design, Envisat is in its minimum drag configuration when Envisat is being towed. In the CDF study, Envisat is in its maximum drag configuration. Referring to Table 1, the surface area of the solar panel is $69.75 \mathrm{~m}^{2}$. The solar panel is oriented at an angle of about $30^{\circ}$, such that in the maximum drag configuration the projected area is about $60.40 \mathrm{~m}^{2}$ and in the minimum drag configuration about $34.87 \mathrm{~m}^{2}$. The surface area of the main body is $27.56 \mathrm{~m}^{2}$ in the maximum drag configuration and $4.4 \mathrm{~m}^{2}$ in the minimum drag configuration. The total projected area in the maximum drag configuration is $87.96 \mathrm{~m}^{2}$. The total projected area in the minimum drag configuration is $39.27 \mathrm{~m}^{2}$. The drag force is given by:

$$
D=q S C_{D}
$$

Where $q=\frac{1}{2} \rho V^{2}$ is the dynamic pressure, $S$ is the area of the spacecraft normal to the flow, $C_{D} \approx 2.2$ is the drag coefficient.

Using this equation leads to an estimate of the drag force experienced by Envisat of $2.763 \mathrm{~N}$ in the maximum drag configuration and $1.2 \mathrm{~N}$ in the minimum drag configuration. The maximum torque is assumed to be equal to the drag force on the solar panel times the moment arm, which is assumed to be equal to $12 \mathrm{~m}$. The maximum drag force on the solar panel is $2.1 \mathrm{~N}$. This means that the maximum torque is equal to $25 \mathrm{Nm}$.

The moment of inertia of Envisat is $129000 \mathrm{~kg} \mathrm{~m}^{2}$, such that this torque causes an angular acceleration of $0.0111^{\circ} / \mathrm{s}^{2}$. Assuming that the drag force acts during a quarter of the orbital period, or $1350 \mathrm{~s}$, and that Envisat remains in its maximum torque configuration, then this torque could lead to an angular velocity of $15 \%$ s. Of course, this is not realistic, because Envisat will not remain in its maximum torque configuration, and the drag force will not be as high as at perigee for one quarter of the orbital period. On the other hand, it does indicate that the attitude and attitude motion of Envisat needs to be kept within certain bounds. Next, the de-orbiting configuration is analysed. Figure 24 shows two proposed capture configuration: (i) ESA's CDF study on the left [8] and the Airbus capture strategy on the right [9]. The main difference between the two is that the ESA configuration assumes that the tether attachment point lies close to the body z-axis of Envisat, whereas the second (Airbus) configuration assumes that the tether attachment point lies close to the body $x$-axis of Envisat. The Airbus configuration has two advantages. First, the moment arm of the tether attachment point has the largest possible moment arm and second, if the chaser remains on V-bar, then the target presents the minimum surface area towards the incoming flow and is also in a configuration that ensures low torque (Envisat's body $x$-axis, meaning the solar panel, would point in the direction of flight). 

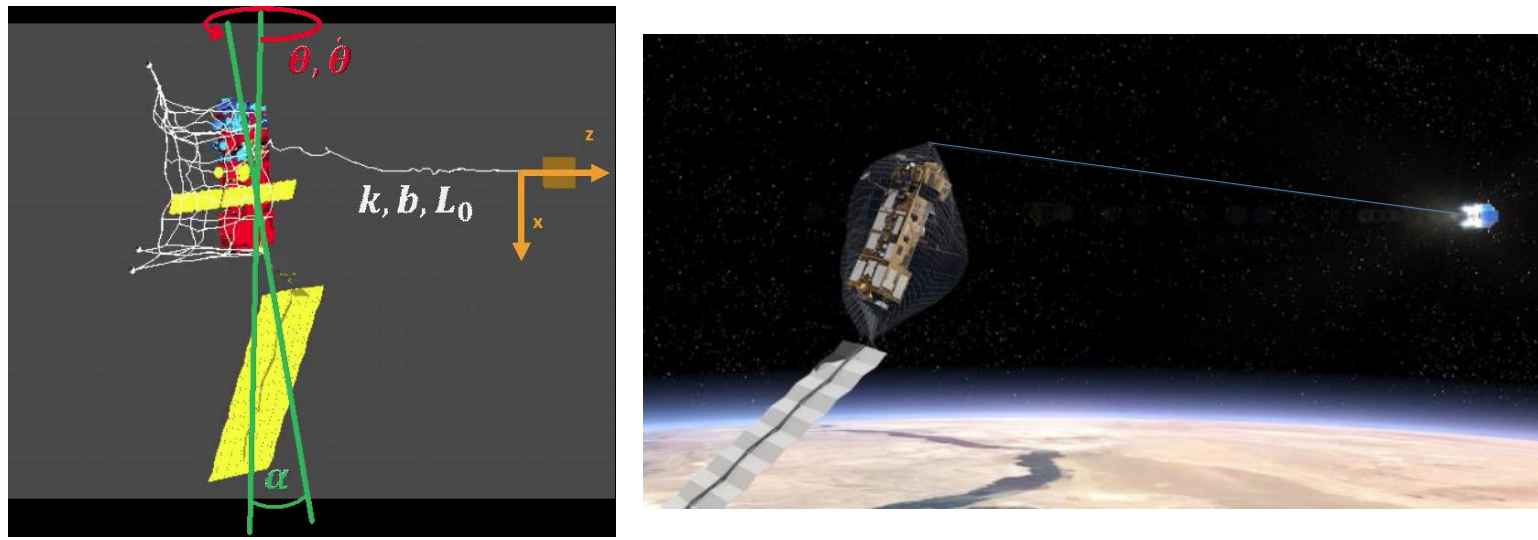

Figure 24: ESA (left) and Airbus (right) capture/tether attachment configurations

In the ESA configuration the moment arm is smaller, and Envisat is in its maximum torque configuration during the pass through the atmosphere. In the Airbus configuration, the moment arm of the tether attachment point has a length of $5 \mathrm{~m}$. In the ESA CDF configuration, the moment arm of the tether attachment point has a length of $1.4 \mathrm{~m}$. The RCS (assist) thrusters create a tether force of about $700 \mathrm{~N}$ in the tether and can therefore impart a torque of $3500 \mathrm{Nm}$ in the Airbus configuration whereas in the ESA CDF configuration a torque of $960 \mathrm{Nm}$ can be generated. Therefore, it can be deduced that, the torques that can be imparted by the chaser on the target, are by far larger than the drag torques. The ADS configuration is easier to control during the atmospheric pass than the ESA CDF configuration, because of the larger moment arm of the tether and due to Envisat being in a low-torque configuration during the atmospheric pass. It is expected that during the atmospheric pass, Envisat, can be brought into a configuration that has a minimum atmospheric drag torque. To reach such an orientation, it is expected that Envisat needs to rotate by at most $90^{\circ}$ (Note that the minimum drag configuration and the maximum drag configuration differ by a rotation of $90^{\circ}$ ). In this case, it can be expected that the atmospheric torque acting on Envisat behaves approximately as follows:

$$
T=T_{\max } \cdot \sin \alpha
$$

\section{Where $\alpha$ is the angle from the minimum drag configuration}

If the maximum angle from the minimum drag configuration is less than $5^{\circ}$, then it can be expected that the atmospheric drag torque acting on Envisat is of the order of $2.2 \mathrm{Nm}$.

\subsubsection{Discussion}

With respect to the stabilization simulations, the proposed thruster configuration ( $220 \mathrm{~N}$ and $22 \mathrm{~N}$ RCS thrusters) is able to cope with the post capture oscillations and the tension output remains well below the maximum tension allowed for Dyneema, an important finding towards selecting tether material for future ADR missions. The peak force required reaches $440 \mathrm{~N}$ which is well within the capability of the four $220 \mathrm{~N}$ thrusters when all of them are used. Fast stabilization for the debris target while in a $5 \% \mathrm{~s}$ initial rate is achieved in less than $20 \mathrm{~s}$ with the alignment angle controlled within $50^{\circ}$, which is crucial to avoid excessive oscillations of the target and potential rupture of the tether. In the case of when only RCS thrusters are used (22N) the alignment angle goes beyond the $90^{\circ}$ from the original orientation and Envisat rotates approximately $100-110^{\circ}$ before it stops rotating. During the maneuver, the thrusters saturate and the risk of tether rupture increase substantially. In simulation cases where lower stiffness tethers are used, it was observed that the desired force on the target is applied with a delay and with lower precision when compared to the nominal stiffness which requires the tether to be extended considerably before it starts delivering the desired force on the target. Therefore, stiffness is an important 
sizing parameter for tethered dynamics and requires detailed analysis with respect to the attitude control forces applied to the overall system. When using lower frequencies, it was observed during stabilization the thrusters are much more active, and that the actuation signals are much noisier, however the tether tension is the same as in the nominal stabilization case simulated, but stays at its peak value substantially longer, thus causing delay in controlling the required attitude angles/rates to zero and eventually drives the system to a slow oscillatory state.

In the de-orbit burn simulations, different tether stiffness models, thrust levels and OBSW frequencies are evaluated and compared with the proposed nominal scenario. In the nominal case where all thrusters are used, tether tension behaves nominally, and the debris pitch and yaw angles are completely damped while the roll attitude enters into a sinusoidal oscillation with a period of approximately $36 \mathrm{~s}$ and within $\pm 6.3^{\circ}$. In scenarios of smaller/assist only thrusters and low frequency, the attitude of the target increases substantially, especially about the roll axis, however it is still controllable and does not become chaotic or dangerous for the chaser.

In summary, the numerical simulations presented in Figures 6-23 show that the proposed control technique can keep the target alignment angle with a controllable and safe range (less than $90^{\circ}$ ) for more stiff tethers, which is due to the increased damping of the target attitude motion caused by the restoring torque generated by the tether tension, thus improving safe towing substantially. The results confirm that for safety reasons, elastic tethers should be avoided, because they can lead to target attitude motion excitation and thus debris collision and fragmentation. It is shown, that the tether damping constant has no significant influence on the target rotation. Furthermore, elastic tethers were discovered to be more difficult to control, due to less tether tension required to maintain the same tether elongation. For stiff tethers, a relatively simple guidance and control system is shown to be sufficient to keep the tether tension to reasonable levels, to stabilize the tether and target dynamics, in a towing mission. In off nominal cases where only attitude control thrusters are available, or the control frequency is low, the proposed tethered controller has been shown to stabilize the tethered system thus avoiding a chaotic or dangerous behavior for the chaser, even when the attitude of the target increases substantially, especially about the roll axis.

When analyzing the stabilization of the debris during an atmospheric pass, tether attachment to the target plays an important role as the torque arm formed by attachment point of the tether, can lead to significant torques which can exceed drag forces substantially, therefore control of the debris attitude to a minimum drag orientation is important.

The analysis from the simulations presented in Figures 6-23 has provided insight on the attitude stability of the tethered system, but also give valuable input on the system design parameters of the tether and control system, for near term ADR towing missions which are discussed below.

\section{a. Simulator structure and tether stiffness}

The analysis and simulations presented so far, clearly indicate that the use of a variable step-size solver is important. If a fixed step is used, then, singularities may occur when simulating stiff tethers with a step size that is too large. The step size needs to be smaller than the natural period of each of the springs:

$$
T_{0, i} \approx 2 \pi \sqrt{\frac{m_{i}}{k_{i}}}
$$

Eq. 21 has a number of implications and consequences for step size and stability:

1) If the discretization is refined, then the mass per element is reduced such that the natural period decreases.

2) The step size needs to be smaller than the natural period of the system.

a. An increase in discretization requires a smaller step size

b. An increase in spring stiffness requires a smaller step size 
c. A decrease in characteristic mass requires a smaller step size

3) If the discretization is refined, then a greater range of frequencies is allowed in the full system

For example, for a $50-m$ tether with overall stiffness $4100 \mathrm{~N} / \mathrm{m}$ and an overall mass of $85 \mathrm{~g}$ that is discretized into 10 point masses, the natural period decreases to 1.3 milliseconds. This is two orders of magnitude below the commonly selected step size of $0.1 \mathrm{~s}$. Singularities occur when a tether mass point moves unrealistically far during the step, leading to unacceptably high forces in the tether. As the natural period becomes shorter than the step size, the simulation can become unstable, that is, the errors in the positions of the point masses can exhibit unbounded growth.

\section{b. Tether stiffness and controllability}

The stiffness of the tether affects the controllability in several ways which should be carefully considered in the design of future ADR missions. The tether stores elastic energy and delays the application of force onto the target. During the initial attitude stabilization, the chaser needs to exert a torque on the target through the tether. In order to exert a torque (i.e., force on the tether attachment point), the chaser would need to move away from its nominal position to put more tension in the tether. If the stiffness is lower, then the chaser needs to move further back. Of course, moving the chaser away from its nominal location takes time, such that the application of force on the target is delayed. The tether stiffness drives the natural frequency of the tether, which may interfere with the frequency of the OBSW. Simulations indicate that an OBSW frequency of $1 \mathrm{~Hz}$ causes the chaser to experience large attitude and position excursions.

\section{c. Use of assist thrusters during initial stabilization}

In the design analysed in this study, the $220 \mathrm{~N}$ thrusters are required to perform the initial stabilization and the stabilization after the de-orbit burn. The assist thrusters can comfortably control the torques imparted by the tether, they can dissipate the rotational kinetic energy after the initial capture and can also dissipate the elastic energy stored in the tether. Both the analysis and simulations presented indicate the need of using the assist thrusters during the initial stabilization, if the target needs to be stabilized before it rotates through $90^{\circ}$.

\section{d. On-Board Software frequency}

Simulation results indicate that the OBSW frequency should be as high as possible. A frequency of $1 \mathrm{~Hz}$ causes large attitude excursions of the chaser. A frequency of $5 \mathrm{~Hz}$ leads to low attitude errors compared to the field of view of typical LIDAR sensors.

\subsection{Conclusion}

In this paper, the capture and towing of a large satellite/debris, Envisat was investigated with respect to its attitude behavior when connected to a chaser with a tether. The attitude motion of debris during tethered towing is investigated, after the debris has been captured by a net. A simple guidance and control system is used and able to keep a small residual tension in the tether, following the deorbit burn. It has been shown that the tether parameters have significant influence on the attitude motion of the target. Careful tether selection and design, reduces the risk of the target rotation excitation even when the step deorbit burn is performed. Numerical simulations show that the proposed control technique can control the target alignment angle within a safe range for more stiff tethers, which is due to the increased damping of the target attitude motion caused by the restoring torque generated by the tether tension, thus improving safe towing substantially. The study confirms that for safety reasons, elastic tethers should be avoided, as they can lead to target attitude motion excitation and thus debris collision and fragmentation. It is shown, that the tether damping constant has no significant influence on the target rotation. Furthermore, elastic tethers are found to be more difficult to control, due to less tether tension required to maintain the same tether elongation. For stiff tethers, a relatively simple guidance and control system is shown to be sufficient to keep the tether tension to reasonable levels, to stabilize the tether and target dynamics, in a towing mission. In off nominal 
cases where only attitude control thrusters are available, or the control frequency is low, the proposed tethered controller has been shown to stabilize the tethered system thus avoiding a chaotic or dangerous behavior for the chaser, even when the attitude of the target increases substantially, especially about the roll axis. The control design and methodology developed in this paper may be useful not only for the stabilization of the tethered system during the coasting phase of active space debris removal missions, but also in the follow-up deorbit burn and stabilization phase, just after net capture of the debris target.

\section{Acknowledgements}

The work presented was funded by the European Space Agency under contract No. 4000116843/16/NL/GLC

\section{References}

[1] Kessler, D. J., Johnson, N. L., Liou, J. C., and Matney, M., "The Kessler Syndrome: Implications to Future Space Operations," 33rd Annual AAS Guidance and Control Conference, AAS Paper 2010-016, Breckenridge, CO, Feb. 2010.

[2] Liou, J. C., "An Active Debris Removal Parametric Study for LEO Environment Remediation," Advances in Space Research, Vol. 47, No. 11, 2011, pp. 1865-1876, doi: 10.1016/j.asr.2011.02.003

[3] Reed, J., and Barraclough, S., "Development of Harpoon System for Capturing Space Debris," 6th European Conference on Space Debris, ESA SP-723, Darmstadt, Germany, April 22 - 25, 2013.

[4] Retat, I., Bischof, B., Starke, J., Froth, W., and Bennell, K., "Net Capture System," 2nd European Workshop on Active Debris Removal, Paper No. 4.3, Quentin, Paris, June 18 - 19 2012.

[5] Reed, J., Busquets, J., and White, C., "Grappling System for Capturing Heavy Space Debris," 2nd European Workshop on Active Debris Removal, Paper No. 4.2, Quentin, Paris, June 18 19, 2012.

[6] Nishida, S., and Yoshikawa, T., "Space Debris Capture by a Joint Compliance Controlled Robot," Proceedings of the 2003 IEEE/ASME International Conference on Advanced Intelligent Mechatronics (AIM 2003), Vol. 1, Kobe, Japan, July $20-24,2003$, pp. 496-502, doi: 10.1109/AIM.2003.1225145

[7] Jasper, L., Schaub, H., Seubert, C., Valery, T., and Yutkin, E., "Tethered Tug for Large Low Earth Orbit Debris Removal," AAS/AIAA Astrodynamics Specialists Conference, Paper No. AAS 12-252, Charleston, SC, January 29 - February 2, 2012.

[8] Jasper, L., Schaub, H., Seubert, C., Valery, T., and Yutkin, E., "Tethered Tug for Large Low Earth Orbit Debris Removal," AAS/AIAA Astrodynamics Specialists Conference, Paper No. AAS 12-252, Charleston, SC, January 29 - February 2, 2012.

[9] Jasper, L., and Schaub, H., "Input Shaped Large Thrust Maneuver with a Tethered Debris Object," Acta Astronautica, Vol. 96, March-April 2014, pp. 128-137.

doi: 10.1016/j.actaastro.2013.11.005

[10] Jasper, L., and Schaub, H., "Tether Design Considerations for Large Thrust Debris De-Orbit Burns," AAS/AIAA Space Flight Mechanics Meeting, Paper No. AAS 14-443, Santa Fe, NM, January $26-30,2014$.

[11] Jasper, L., and Schaub, H., "Tethered Towing Using Open-Loop Input-Shaping and Discrete Thrust Levels," Acta Astronautica, Vol. 105, No. 1, Dec. 2014, pp. 373-384.

doi: 10.1016/j.actaastro.2014.10.001

[12] Jasper, L., "Open-Loop Thrust Profile Development for Tethered Towing of Large Space Objects," Ph.D. Thesis, Aerospace Engineering Sciences Dept., Univ. of Colorado, Boulder, CO, 2014. 
[13] Linskens, H. T. K., and Mooij, E., "Tether Dynamics Analysis and Guidance and Control Design for Active Space-Debris Removal," Journal of Guidance, Control and Dynamics, Vol. 39, No. 6, 2016, pp. 1232-1243.

doi: 10.2514/1.G001651

[14] Kucharski, D., Kirchner, G., Koidl, F., Fan, C., Carman, R., Moore, C., Feng, Q., 2014, “Attitude and Spin Period of Space Debris Envisat Measured by Satellite Laser Ranging", IEEE Transactions on Geoscience and Remote Sensing, Vol. 52, Issue 12, pp. 7651 - 7657, DOI: 10.1109/TGRS.2014.2316138, 2014

[15] Aslanov, V. S., and Yudintsev, V. V., "Dynamics of Large Space Debris Removal Using Tethered Space Tug," Acta Astronautica, Vol. 91, Oct.-Nov. 2013, pp. 149-156, doi: 10.1016/j.actaastro.2013.05.020

[16] Aslanov, V. S., and Ledkov, A. S., "Dynamics of Towed Large Space Debris Taking Into Account Atmospheric Disturbance," Acta Mechanica, Vol. 225, No. 9, 2014, pp. 2685-2697. doi: 10.1007/s00707-014-1094-4.

[17] Biesbroek, R., Hüsing, J., and Wolahan, A., "System and Concurrent Engineering for the eDeorbit Mission Assessment Studies," 6th International Systems and Concurrent Engineering for Space Applications Conference, Stuttgart, Germany, Oct. 2014.

[18] Gómez, N. O., and Walker, S. J. I., "Earth's Gravity Gradient and Eddy Currents Effects on the Rotational Dynamics of Space Debris Objects: Envisat Case Study," Advances in Space Research, Vol. 56, No. 3, August 2015, pp. 494-508, doi: 10.1016/j.asr.2014.12.031

[19] Kucharski, D., Kirchner, G., Koidl, F., Fan, C., Carman, R., Moore, C., et al., "Attitude and Spin Period of Space Debris Envisat Measured by Satellite Laser Ranging," IEEE Transactions on Geoscience and Remote Sensing, Vol. 52, No. 12, Dec. 2014, pp. 7651-7657, doi: 10.1109/TGRS.2014.2316138

[20] CDF, "e.deorbit CDF study report CDF", CDF-135(C), Sep 2012

[21] Biesbroek, R., Hüsing, J., and Wolahan, A., "System and Concurrent Engineering for the eDeorbit Mission Assessment Studies," 6th International Systems and Concurrent Engineering for Space Applications Conference, Stuttgart, Germany, Oct. 2014.

[22] Falcoz, A. Moro , V., 2014, "AGADiR - Advanced GNC for Active Debris Removal," e.Deorbit Symposium: 06/05/2014 - Noordwijkerhout, The Netherlands, 06/05/2014

[23] Michiel Kruijff, Erik van der Heide, Wubbo Ockels, and Eberhard Gill. "First Mission Results of the YES2 Tethered SpaceMail Experiment", AIAA/AAS Astrodynamics Specialist Conference and Exhibit, Guidance, Navigation, and Control and Co-located Conferences, DOI: 10.2514/6.2008-7385, 2008

[24] Michiel Kruijff, "Tethers in Space," PhD thesis, TU Delft, 2011

[25] B. Bastida Virgili, S. Lemmens, H. Krag, Investigation on Envisat attitude motion, e.Deorbit Workshop, 2014

[26] Airbus Defence and Space, "Space Propulsion - Chemical Bi-Propellant Thruster Family 4N, $10 \mathrm{~N}, 22 \mathrm{~N}, 200 \mathrm{~N}, 400 \mathrm{~N}$ ", Data Sheet, 2013

[27] F. Zhang and P. Huang, "Releasing Dynamics and Stability Control of Maneuverable Tethered Space Net," in IEEE/ASME Transactions on Mechatronics, vol. 22, no. 2, pp. 983-993, April 2017. doi: 10.1109/TMECH.2016.2628052

[28] Huang, P., Wang, D., Zhang, F., Meng, Z., and Liu, Z., 'Postcapture robust nonlinear control for tethered space robot with constraints on actuator and velocity of space tether'. International Journal of Robust Nonlinear Control, 27: 2824-284, 2017. doi: 10.1002/rnc.3712.

[29] Minghe Shan, Jian Guo, Eberhard Gill, 'Contact dynamic models of space debris capturing using a net', Acta Astronautica, 2017, ISSN 0094-5765, https://doi.org/10.1016/j.actaastro.2017.12.009 
[30] Yakun Zhao, Panfeng Huang, Fan Zhang, 'Dynamic modeling and Super-Twisting Sliding Mode Control for Tethered Space Robot', Acta Astronautica, Volume 143, 2018, Pages 310-321, ISSN 0094-5765, https://doi.org/10.1016/j.actaastro.2017.11.025

[31] Bingheng Wang, Zhongjie Meng, Panfeng Huang, Attitude control of towed space debris using only tether, Acta Astronautica, Volume 138, 2017, Pages 152-167, ISSN 0094-5765, https://doi.org/10.1016/j.actaastro.2017.05.012

[32] Jingrui Zhang, Keying Yang, Rui Qi, 'Dynamics and offset control of tethered space-tug system', Acta Astronautica, Volume 142, 2018, Pages 232-252, ISSN 0094-5765, https://doi.org/10.1016/j.actaastro.2017.10.020

[33] P. Huang, F. Zhang, J. Cai, D. Wang, Z. Meng and J. Guo, "Dexterous Tethered Space Robot: Design, Measurement, Control, and Experiment," in IEEE Transactions on Aerospace and Electronic Systems, vol. 53, no. 3, pp. 1452-1468, June 2017, doi: 10.1109/TAES.2017.2671558 
2018-03-12

\title{
Attitude control analysis of tethered de-orbiting
}

\author{
Peters, T. V.
}

Peters TV, Valero JF, Lappas V, et al., Attitude control analysis of tethered de-orbiting. Acta Astronautica, Volume 146, May 2018, pp. 316-331

https://doi.org/10.1016/j.actaastro.2018.03.016

Downloaded from Cranfield Library Services E-Repository 